Análisis y Modificación de Conducta, 2006, Vol. 32, № 144

\title{
INTERESES, CONDUCTA SEXUAL Y COMPORTAMIENTOS DE RIESGO PARA LA SALUD SEXUAL DE ESCOLARES ADOLESCENTES PARTICIPANTES EN UN PROGRAMA DE EDUCACIÓN SEXUAL
}

\author{
Ángeles Palenzuela Sánchez \\ Centro de Salud Sexual y Reproductiva. Conselleria de Sanidad. Generalitat Valenciana. \\ Alicante
}

\section{RESUMEN}

El presente trabajo constituye un estudio descriptivo mediante encuesta semiestructurada en una muestra de 501 escolares adolescentes de secundaria participantes en un programa de educación sexual. Se pretende: describir la frecuencia y distribución de las conductas sexuales más frecuentes en escolares adolescentes participantes en el programa, grado de información y conocimientos en sexualidad $y$ sus fuentes; creencias, actitudes y mitos en sexualidad; intereses y demandas de información; conocer diferentes factores que conllevan conductas de riesgo en sexualidad en los adolescentes en relación a embarazos no deseados y E.T.S: déficits en información sexual, creencias erróneas $y$ actividad sexual. Los resultados muestran que el $47,70 \%$ de los adolescentes encuestados reconocian haber tenido algún tipo de actividad sexual: un $36,32 \%$ de la muestra realizan conductas autoestimulatorias, un $41,51 \%$ caricias y besos, $y$ un $3,39 \%$ 
realizan actividad sexual coital. Los varones iniciaron la actividad sexual antes que las mujeres y en cualquier manifestación de las mismas y el porcentaje que manifiesta llevarlo a cabo supera al de las mujeres. Un $40,31 \%$ desconocen que son los M.A., y un $37,31 \%$ no sabrian usar adecuadamente un preservativo. Un $68,25 \%$ no saben la ubicación del clítoris y un $59,09 \%$ no saben si el mismo puede producir placer en la mujer. Un $29,52 \%$ mantienen creencias erróneas y mitos sobre la masturbación y un $30,52 \%$ en torno a la homosexualidad. Los adolescentes demandan información en primer lugar de las relaciones sexuales, y desconocen la existencia de los recursos sanitarios en esta materia. Los adolescentes valoran positivamente intervenciones educativas a nivel de sexualidad.

En conclusión, un elevado porcentaje de adolescentes manifiestan deficiencias en la información sexual recibida, presentan bajos conocimientos y creencias erróneas en materia de sexualidad, y déficits de información en M.A. y es una población que presenta actividad sexual. Consideramos que estos factores son conductas de riesgo para su salud sexual y reproductiva tanto en su presente inmediato como en futuro próximo con consecuencias para su salud sexual, tales como: E.M.N.D., E.T.S. y Sida, I.V.E., maternidad prematura o disfunciones sexuales. Se valora las implicaciones de estos resultados obtenidos en relación a la relevancia de implantar programas de educación sexual a escolares adolescentes a edades tempranas. El objetivo final sería la capacitación y adopción de comportamientos que promuevan la preservación de su salud sexual, y asi poder planear su vida sexual futura y, por tanto, mejorar su calidad de vida.

Palabras clave: COMPORTAMIENTO SEXUAL. ADOLESCENCIA. INFORMACIÓN SEXUAL. EDUCACIÓN SEXUAL.

\section{SUMMARY}

A sample of 501 teenagers (secondary school) fulfilled a half structured survey, participating in a program of sexual 
education. The objectives are: 1 - to describe the frequency and distribution of sexual behaviours in these students, their level and sources of information and sexual knowledge, convictions, thoughts and myths in sexuality; interests and requests for information; and 2- to know the different factors which suppose risky conducts in the adolescents sexuality as undesired pregnancies and deficits in sexual information, wrong assumptions and sexual activity. The results show that a $47.70 \%$ of the interviewed teenagers have had any kind of sexual activity, a $36.32 \%$ stimulate themselves, a $41.51 \%$ caresses and kisses and a 3.39\% have coital relationships. Men start sexual activity before than women in any case and the percentage of realization is also higher. A $40.31 \%$ do not know what the contraceptive methods are and a $37.31 \%$ will not be able to use a preservative properly. A $68.25 \%$ do not know the location of the clitoris and a $59.09 \%$ do not know if it can produce pleasure in women. A29.52\% have wrong assumptions and myths regarding masturbation and a $30.52 \%$ regarding homosexuality. Teenagers ask for information firstly about the sexual relations and ignore the available sanitary resources. Teenagers value interventions in sexual education. In conclusion, a high percentage of teenagers - with sexual activity- manifest having received deficient sexual information and present a low knowledge and wrong assumptions in sexuality and lack of information in contraceptive methods. We think that these factors present a risky condition both sexual and reproductive, both in the present and in the future having consequences as preventing unwanted pregnancies, sexually transmitted diseases and AIDS, voluntary interruption of pregnancy, premature maternity or sexual dysfunctions. We value the implications of these results with he relevance of new programs of sexual education to youngest scholars. The final objective would be the capacity and adoption of behaviours which promote the preservation of their sexual condition and then the capacity of planning a better, safer and more qualified future life.

Key words: SEXUAL BEHAVOUR. ADOLESCENCE. SEXUAL INFORMATION. SEXUAL EDUCATION. 


\section{INTRODUCCIÓN}

La adolescencia supone un periodo de la vida clave en el desarrollo de cualquier individuo, constituyendo una etapa de transición que va desde el final de la infancia hasta el inicio de la vida adulta (Palau, 1992; Erikson, 1971, 1993). Es un periodo de importantes modificaciones desde el punto de vista físico, psíquico, y social (Pick, Givaudan y Saldívar-Garduño, 1994), adquiridas evolutivamente a lo largo de las diferentes edades que conforman la adolescencia, considerándose esta etapa como un proceso. Es el periodo de las "operaciones formales" según Piaget y ello conlleva que los adolescentes "empiezan a tener capacidad de pensar de manera abstracta, sus razonamientos son más elaborados" (Guerra, 1999:83).

La Organización Mundial de la Salud (O.M.S.) define la misma como "el periodo de la vida en el cual el individuo adquiere la capacidad reproductiva, transita los patrones psicológicos de la niñez a la adultez y consolida su independencia económica" (OPS/OMS, 1995), fija sus límites entre los 10-19 años y los de la juventud entre los 15 y 24 años (Ruiz, Almenara, Rodríguez y Fernández, 1990; A picture of health? A review and annotated bibliography of the health of young people in developing countries, 1995). Distingue una adolescencia de primer tramo -que va hasta los 16 años llamada de baja edad y la de mayor riesgo, y otra, de segundo tramo, a partir de los 16 años llamada de alta edad-.Por su parte, Guerra (1999) distingue una adolescencia temprana (10-13 años), una media (14-16 años) y otra tardía (17-19 años); $y$ otros autores toman como juventud la adolescencia media y tardía (Stassen y Thompson, 2000). Debido a este solapamiento de edades según las diferentes definiciones, muchos de los estudios acerca del comportamiento sexual en la adolescencia podrian no ser comparables. Por ello conviene pensar de manera puntual el objeto de estudio que ocupa nuestra investigación: se trata de la adolescencia temprana y media, o del primer tramo según la OMS. Este periodo se caracteriza desde un punto de vista psicológico entre otros: por aumentar las habilidades cognitivas, falta de control de impulsos, necesidad de independencia, máxima conflictividad con los padres, conformidad con los valores del grupo, sentimientos de invulnerabilidad que les lleva a realizar conductas de riesgo, les preocupa los cambios 
producidos en la pubertad, su apariencia física, deseo de una imagen corporal atractiva (Guerra, 1999). Como puede comprenderse, se trata de una etapa de cambios físicos y psicosociales cruciales, se producen las primeras manifestaciones y comportamientos sexuales y con éstos "se instauran actitudes y hábitos de conducta» (Lameiras, Rodríguez, Calado y González, 2004:68). Los impulsos sexuales son elevados, el contacto físico se hace más urgente y predominante y la conducta sexual aparece como aventura y exploratoria (Gascón, Navarro, Gascón, Pérula de Torres, Jurado y Montes, 2003b). La madurez biofisiologica se adquiere antes que la psicosocial, por lo que la mayoría no han madurado o desarrollado sus capacidades y habilidades para afrontar situaciones diversas, lo que unido a la baja percepción del riesgo de los adolescentes (Fisher, 1988) puede tener graves repercusiones en su salud y entre ellas para su salud sexual. La morbimortalidad en los adolescentes es baja pero no están exentos a factores de riesgo a la salud en general (tabaquismo, accidentes, alcoholismo, sobrepeso, etc.; (OPS/OMS, 1998). Además, en esta etapa eclosiona su sexualidad y por tanto los riesgos para su salud sexual: embarazos no deseados (E.M.N.D.), enfermedades de transmisión sexual (E.T.S.) y sida, interrupción voluntaria del embarazo (I.V.E.), maternidad prematura, disfunción sexual futura, etc.

Para algunos adolescentes los comportamientos de riesgo no son más que una experimentación, y para otros, sin embargo son comportamientos que desarrollan habitualmente, persistiendo en la etapa adulta, con los riesgos que ello entraña para la salud en general y la salud sexual en particular. Mendoza, Sagrera y Batista-Foguet (1994) refieren que tanto la salud en general como la sexual dependen de los estilos de vida de la persona, de sus patrones de conducta que caracterizan su manera de vivir, y reseñan que en el grupo de edad de 15-25 años las conductas de riesgos se incrementan en exceso. Tal y como indican Millstein, Petersen y Nightingale (1993), muchos de los comportamientos que se asocian a la morbimortalidad en adultos se inician en esta época. Al valorar los estudios sobre la sexualidad de los adolescentes vemos que se han enfocado desde dos perspectivas opuestas; a saber, la positiva, que "estudia la influencia de las relaciones afectivo-sexuales en el desarrollo de la personalidad y y la negativa, que estudia la influencia de los riesgos de la salud sexual en 
los adolescentes, vinculando la actividad sexual al incremento de los factores de riesgo (Lameiras «et al.», 2004:68). Esta última perspectiva es el objetivo de nuestro estudio, que podemos concretar en la valoración de los excesos o déficits comportamentales que pueden afectar a nuestra salud sexual; en definitiva, los comportamientos sexuales de riesgo para la salud sexual en esta etapa de la vida donde las conductas y actitudes adquiridas influirán y guiaran nuestra adultez.

La O.M.S., entre otros organismos, considera a la población adolescente como una población de riesgo en materia de sexualidad y reproducción, y considera a éstos como un grupo prioritario en materia de intervenciones en el nivel de la prevención, y sobre todo en el nivel educativo. De las diferentes investigaciones al respecto se deduce que los adolescentes presentan conductas de riego para su salud sexual (en relación a E.M.N.D., E.T.S. y Sida, I.V.E.) (Martínez, 2000). Estas consecuencias para su salud sexual han ido aumentando progresivamente, lo que podría ser debido a diferentes causas como: el inicio de actividad sexual a edades cada vez más tempranas (Martín y Velarde, 1996), déficits de conocimientos en sexualidad, baja percepción del riesgo en los adolescentes, maduración biológica más temprana (adelanto de la menarquia), la asociación con otros comportamientos de riesgo como el consumo de alcohol asociado a hábitos sexuales (Mott, Fondell, Hu, Kowaleski-Jones y Menaghan, 1996; Lauchli, Heussr, Tschopp y Gutzwiller, 1996; O'Hara, Parris, Fichtner y Oster, 1998), o también al hecho de que las primeras relaciones sexuales son generalmente espontáneas y no previstas, lo que dificulta el uso de un método anticonceptivo (López, 1995; Loewenstein y Furstenberg, 1991).

Hay que partir del hecho que los adolescentes son activos sexualmente y la mayoría de ellos han tenido experiencias sexuales personales o interpersonales. Martínez (2000) refiere que la conducta heterosexual se produce respecto a otros años a edades cada vez más tempranas. En algunos estudios se concluye que en la adolescencia temprana se darían los primeros contactos sexuales y que en la media-tardía la mayor parte de los adolescentes tendrían su primera relación sexual coital (García, 1994; Malo de Molina, 1992; Oliva, Serra y Vallejo, 1992). Según datos del Instituto de la Juventud (INJUVE) en el informe del 2004 de la Juventud en España (Aguinaga, 
Andréu, Cachón, Comas, López, y Navarrete, 2005) se refiere que en los adolescentes de $15-17$ años el $28,3 \%$ habían tenido relaciones sexuales coitales y en el grupo de $18-20$ años el $75,3 \%$. Concluyen que a nivel de experiencia sexual en los adolescentes de 15-17 años ha aumentado su frecuencia, duplicándose en número desde el año 2000 al 2004 en ambos sexos. Ramos, Fuertes, Martínez y Hemández (2003) concluyen de la muestra de adolescentes estudiada que la mayoría habian tenido algún tipo de experiencia heterosexual no coital y un $18,6 \%$ de los mismos relación sexual coital. Resultado parecido encontramos en Gascón»et al» (2003b) donde se nos dice que un $22,9 \%$ de la muestra reconocen tener relaciones sexuales completas.

En España, la incidencia de E.M.N.D. y E.T.S. ha aumentado a pesar de las campañas de información (Romero de Castilla, Lora y Cañete, 2001). En relación a los E.M.N.D. en la adolescencia, según los datos del Consejo Superior de Investigaciones Científicas (C.S.I.C.) en el grupo de edad de entre 15-19 años la tasa de embarazo en el año 2000 era de 16,21 por 1000 mujeres, en el 2001 se situaba en el 17,92, en el 2003 la tasa se sitúa en el 20,31 y en el 2004 se sitúa en el 21,20 . Observamos, pues, que se incrementan las tasas ligeramente pero de forma ascendente. Esta situación conlleva importantes consecuencias para la salud en general y la sexual en particular. Los embarazos en <20 años presentan el doble de riesgos biológicos, además de los riesgos socio-culturales de estos casos y también para el recién nacido (Valerio, 1985; Portella y Castillo, 1988; Vallés, 1986). En los datos obtenidos de la encuesta Daphne (2003) sobre la utilización de los métodos anticonceptivos (M.A.) en España, se concluye que en el grupo de edad de 15-19 años un 54,8\% no usaron ningún tipo de método. Este dato es relevante si lo asociamos a los datos de diferentes estudios que sitúan la edad de inicio de relaciones sexuales sobre los 16 años (Gascón»et al.», 2003b) y en otros, como en el informe de la juventud en España del 2004 que manifiesta que en varones de 15-17 años que mantienen relación sexual un $13,5 \%$ no usan el preservativo y el $66,8 \%$ dan como motivo de ello el no tenerlo a mano, prefiriendo exponerse al riesgo en lugar de abstenerse de una relación sexual sin protección. La consecuencia más relevante de los E.M.N.D. por su frecuencia sería las I.V.E. Según Delgado (2003), de las adolescentes que quedan embarazadas un 
$44 \%$ recurren a I.V.E. y respecto a las tasas de I.V.E. crecientes en las mismas, señala las deficiencias en el uso de método anticonceptivo en las adolescentes. Según los datos del Ministerio de Sanidad y Consumo en los informes sobre la I.V.E. en España, en $<19$ años la tasa específica de abortividad por 1000 mujeres se situaba en 8,29 en el año 2001, en el 2002 en un 9,28, en el 2003 en un 9,90, y en el 2004 en un 10.57 registrándose en este grupo de edad el mayor aumento de I.V.E. con respecto al año anterior. Se pone de manifiesto una tendencia ascendente en las tasas de abortividad, de un 4,22 en 1994 hasta el 10,57 en el 2004. También sube ligeramente los abortos de repetición. En la Comunidad Valenciana, para este mismo grupo de edad, según datos del Ministerio de Sanidad y Consumo las tasas de embarazo se sitúan desde un 15,83 en el año 2000 , un 18,52 en el 2002 , el 19,43 en el 2003 y hasta en el 2004 un 20,54. Respecto a la I.V.E., según los datos de la Conselleria de Sanidad de la Generalitat Valenciana, en la Serie informes de salud: Interrupciones voluntarias del embarazo en la C.V. 2004 y en el grupo de $<20$ años, experimentan un aumento en el 2004 respecto a los años anteriores, volviéndose a porcentajes alcanzados en el año 2000 , en el 2004 se sitúa en un $14,1 \%$ y en el 2003 el $13 ; 4 \%$.

Respecto a las E.T.S. y Sida, también los adolescentes están expuestos ya que muchos de ellos carecen de información necesaria y adecuada de los riesgos de sus conductas sexuales y de cómo protegerse adecuadamente de los mismos. Ammerman, Perelli y Adler (1992) concluyen que existe un gran déficit de conocimientos sobre sexualidad y reproducción en los adolescentes de su estudio. La falta de información sobre las E.T.S. perpetúan creencias e ideas erróneas sobre estas enfermedades, lo que lleva a una estigmatización de las mismas que trae como consecuencia la no atención sanitaria y en ausencia de síntomas de las mismas la no atención sanitaria oportuna y adecuada, con importantes consecuencias para su salud tanto física como reproductora. Moscoso, Rodríguez, Parrilla, Robles y Colón (1997) en el estudio realizado sobre cinco factores de riesgo frente al V.I.H. concluyen como resultado que el $47,9 \%$ de los adolescentes tenían dos o más factores de riesgo. Otros estudios asocian la edad de inicio de relaciones sexuales cada vez más tempranas a un mayor riesgo de E.T.S. (Baldwin, Wills y Link, 1998; Levy, Handler y Weeks, 
1995) y E.M.N.D. (Allen, 1984; Melchert y Burnett, 1990). En el informe sobre la epidemia mundial del $\mathrm{VIH} /$ sida ONU/OMS del 2002, se refiere que más del $50 \%$ de las nuevas infecciones por V.I.H. se produjeron en el grupo de adolescentes de 10-24 años (Onusida, 2002). En España, según el INJUVE, en el año 2001, en el grupo de 15-19 años, el $2,7 \%$ de varones y el $5,9 \%$ de mujeres, los contagios se produjeron por relaciones heterosexuales de riesgo. En el informe de la Juventud en España 2004 (Aguinaga «et al.», 2005), se refiere que las tasas de sida en el mismo grupo anterior representan el 30,1 de los casos. Respecto a los conocimientos de conductas preventivas frente a E.T.S. y sida, se refiere que el $93 \%$ de jóvenes que usaron el preservativo en la relación sexual fue motivado para prevenir un embarazo y en menor medida para protegerse del sida (el $34,8 \%$ ) o E.T.S. (el $31,9 \%$ ), por lo que se concluye que esta información no está bien asimilada entre los jóvenes.

Partiendo de estos resultados y de los diferentes factores que contribuyen a incrementar los riesgos para la salud sexual de los adolescentes, nos planteamos como objetivo específico de nuestro trabajo valorar en primer lugar qué situación en el nivel de la sexualidad y la reproducción tenian los adolescentes situados en nuestra área de salud para adecuar nuestra intervención educativa en temas de promoción de la salud sexual de los adolescentes. Diferentes estudios (Valerio, 1985; Howard, 1985; Ehrhardt, Yingling, Zawadski y Martínez, 1992; Kirby, 1992) revelan la importancia de los programas de educación sexual para reducir los factores de riesgos anteriormente estudiados, E.M.N.D. y E.T.S. Otros estudios que apoyan este tipo de intervenciones con los adolescentes lo encontramos en Barros uet al.» (2001); Yamada, Herrera, Cota, Núñez y Guzmán (2002). Sin embargo, esta información y educación sexual no sólo tiene como objetivo la prevención de estos riesgos, sino que ha de valorarse en un contexto más amplio de educación para la salud "comprendida como la creación de hábitos de higiene física y mental que permitan un desarrollo personal saludable, una aceptación del propio cuerpo, y un respeto hacia las actitudes y comportamiento sexual de los demás» (Bethencourt, Fumero, Fernández y León, 1997:236) y también con la perspectiva de salud sexual definida por la O.M.S. en el 2002 «como un estado de bienestar físico, emocional, mental y social relacionada 
con la sexualidad; no es meramente la ausencia de enfermedad, disfunción o debilidad. La salud sexual requiere un acercamiento positivo y respetuoso hacia la sexualidad y las relaciones sexuales, así como hacia la posibilidad de obtener placer y hacia las experiencias sexuales seguras, libres de coerción, discriminación y violencia. Para que la salud sexual se logre y se mantenga, los derechos sexuales de las personas deben ser protegidos y cumplidos" (Franco y Landaarroitajauregui, 2005:98). En la Conferencia Internacional sobre Población y Desarrollo, celebrada en El Cairo en 1994, se realizó una serie de recomendaciones que mejoraran el acceso a los servicios de salud reproductiva por los adolescentes, produciéndose un cambio en el concepto de planificación familiar que debiera entenderse como «salud reproductiva» significando la misma como «el hecho de llevar una vida sexual responsable, satisfactoria y segura, además de la capacidad de reproducirse y decidir libremente, cuándo, cómo y cuánto" (Lete y Martínez-Etayo, 2004:170). La definición de salud reproductiva que se aprueba en esta conferencia y propuesta por la O.M.S. incluye la salud sexual (Population and Development, I: Programme of Action adopted at the International Conference on Population and Development, 1994).

El estar adecuadamente informados es necesario para eliminar creencias equivocadas (Valerio, 1985) lo que es de gran importancia para la manera en que se vivencia la sexualidad y el origen de las disfunciones sexuales, ya que muchas de las mismas están causadas por desconocimientos o creencias erróneas en torno al hecho sexual, vivenciándose la misma con vergūenza o culpa.

Por todo lo dicho, podemos concretar que el presente estudio descriptivo tiene como objetivo final, en primer lugar, conocer las diferentes conductas de riesgo a su salud sexual de nuestra muestra de adolescentes objeto del programa de educación sexual, tanto en el nivel de experiencia sexual y edades de inicio como en el nivel de información, conocimientos, creencias erróneas en torno a la sexualidad; asi como, en segundo lugar, recoger sus demandas de información y campos de interés con el objeto de ajustar el programa de educación sexual a sus necesidades; $y$, en tercer lugar, valorar las diferencias entre los sexos y conocer la opinión de los adolescentes respecto a la intervención recibida como método para mejorar su 
formación personal en sexualidad. Estos datos se recogieron con la intención de ser un punto de partida para desarrollar estrategias de intervención encaminadas a la promoción de salud sexual en los adolescentes de nuestra área de salud, tanto desde un punto de vista de prevención de riesgos para la salud sexual, como desde otra visión más amplia de promoción de salud sexual, vivenciándola como saludable y placentera, como forma también de prevenir futuras disfunciones sexuales, teniendo en cuenta que una de las causas de las mismas son los factores personales, y entre ellos, la falta de información sexual, inadecuada educación sexual, miedos, o mitos culturales irracionales (Masters, Johnson y Kolodny, 1987). Siguiendo a Ramos y Cantú (2003) estamos de acuerdo en que estos programas no sólo ofrezcan información para la prevención sino que también se orienten a fomentar valores positivos que den como resultado una vida sexual más segura y con asunción de responsabilidades.

\section{MÉTODO}

\section{Sujetos y diseño}

La presente investigación se ha realizado sobre una muestra de 501 escolares adolescentes de ambos sexos, de los cuales 227 $(45,30 \%)$ eran mujeres y $274(54,69 \%)$ eran varones. La encuesta fue administrada en un rango de edad comprendido entre los 12 y 16 años con una media de edad de 13,3 años y D.T $=1,57$. El 1,59\% de la muestra estuvo compuesto por adolescentes de 12 años, el $68,66 \%$ por adolescentes de 13 años, el 23,35\% por escolares de 14 años, el $5,58 \%$ de 15 años y por último un $0,79 \%$ por adolescentes de 16 años. La muestra estaba compuesta por escolares que cursaban en ese momento $2^{2}$ de ESO y escolarizados en centros públicos y concertados que aceptaron participar en un programa de educación sexual y perteneciente al área de salud de un Centro de Orientación y Planificación Familiar (C.O.P.F.) de Alicante, dependiente de la Consellería de Sanidad. La muestra pertenecía a 11 colegios entre públicos y concertados de los 19 que abarcaba nuestra área de salud. Los participantes respondieron a la encuesta de forma anónima y voluntaria en horario lectivo. 
El diseño se corresponde con un estudio descriptivo mediante encuestas con diseño transversal (Montero y León, 2002).

\section{Instrumentos y variables}

Para este estudio el instrumento utilizado fue una encuesta descriptiva semiestructurada elaborada ad hoc según los contenidos del programa de educación sexual que los alumnos iban a recibir y basada en cuestionarios previos extraídos de la bibliografía existente (Ras, Lluis, Subirats, Pellejo, Lara y Rodríguez, 2004; Rebull, Reverté, Piñas, Ortí, González y Contreras, 2003; Gascón, Navarro, Gascón, Pérula, Jurado y Montes, 2003a; Oliva, Serra y Vallejo, 1993; Mendoza «et al.», 1994; López, 1995; García, 1990). La encuesta incluía 25 items, de los que 22 cuestiones tratadas eran de respuesta cerrada dicotómica o categorías de respuesta limitada alguna de ellas, y 3 de las cuestiones, de respuesta abierta para recoger básicamente intereses y demandas de información que posteriormente han sido agrupadas y categorizadas para análisis posterior en bloques temáticos. Las cuestiones eran de acción, de información, y opinión con el objeto de valorar conocimientos, información sexual, opiniones y actitudes ante el hecho sexual, actividad sexual e intereses y demandas de información. Se realizó también una valoración cualitativa de la intervención educativa con los adolescentes. Para ello, respondieron a otra encuesta de opinión estructurada de la que 16 ítems eran de respuesta dicotómica o categorías de respuesta limitada y 6 ítems eran de respuesta abierta para valorar la percepción, utilidad y opinión de los adolescentes acerca del programa de educación sexual. Dos de las mismas se eligen con este formato con el fin de que los adolescentes fueran capaces de evocar espontáneamente la respuesta más que reconocerla, valorando así dos de las conductas más importantes relacionadas con los riesgos para su salud sexual. El objetivo era valorar aspectos formales, temas, dudas principales, cómo lo vivencian, mejoras, etc. para tenerlo presente en futuras intervenciones.

Mediante la encuesta autoadministrada, anónima, voluntaria y previa información de los objetivos de la misma y de las normas para su cumplimentación, se recogieron datos sobre la sexualidad de los 
adolescentes. Las variables a evaluar fueron: sexo y edad; conocimientos $\theta$ información en sexualidad y fuentes de información; conocimientos sobre M.A. y E.T.S.; creencias, actitudes y mitos sexuales; nivel de actividad sexual y edad de inicio de las mismas; intereses y demandas en sexualidad; opinión y valoración por los adolescentes de una intervención educativa en su formación sexual.

\section{Procedimiento y Análisis de datos}

El análisis de datos ha consistido en un análisis descriptivo para obtener frecuencias simples de cada una de las variables del estudio y su distribución. Se describen las variables cualitativas y cuantitativas. Los análisis realizados consistieron en la obtención de frecuencias y porcentajes. Para explorar posibles diferencias significativas de género se aplica la prueba Chi cuadrado. Para las preguntas abiertas se hace el agrupamiento de todas las respuestas y se cuantifican porcentualmente. El procedimiento de análisis de datos se ha centrado en torno a: grado de información y fuentes, conocimientos y actitudes sexuales, conocimientos de M.A. y E.T.S., conducta sexual que manifiestan en estas edades, intereses de los adolescentes en materia de sexualidad.

La obtención de los datos de la encuesta a los escolares de secundaría formaba parte de la aplicación de un programa de promoción de salud sexual desde un C.O.P.F. y su área de salud.

Se propuso un programa de promoción de salud sexual a los centros educativos que constituían la zona de influencia del área de salud dónde pertenecía el C.O.P.F., dirigido a estudiantes de $2^{2}$ de E.S.O. Se contactó por carta informativa con los centros educativos pertenecientes a nuestra área de salud, ofreciéndoles la posibilidad de realización del programa de educación sexual. Por cada centro educativo que contestaba estar interesado en el mismo se hizo una reunión informativa con la dirección, profesorado, APA, para exponerles los objetivos, contenidos y organización del mismo y llegar a acuerdos de colaboración. Previo a la implantación del programa a los alumnos, se convocó también a los padres a una sesión informativa-formativa para conseguir su aceptación y colaboración, manifestando en la misma convocatoria escrita la aceptación o rechazo a que su hijo 
recibiera esta intervención educativa. Una vez aceptada su aplicación en el centro educativo, a los alumnos se les explicaba que recibirian un programa de educación sexual al que previamente se le solicitaba su colaboración en la cumplimentación de una encuesta de carácter totalmente voluntaria y anónima que exploraba sus conocimientos y actividad sexual. Se insistía en el anonimato, voluntariedad y en contestar lo más sinceramente posible; estas instrucciones constaban por escrito en el encabezado de la encuesta y se les agradecia su colaboración.

Las encuestas se administraron de forma colectiva y en horario lectivo antes de la intervención educativa con el objeto de ajustar lo máximo posible los contenidos del programa a las demandas y necesidades de los encuestados.

El desarrollo del programa consistió en cinco ciclos temáticos de trabajo directo con el alumnado de una hora y media de duración cada uno de ellos, periodicidad semanal, en horario lectivo y en los grupos naturales de las aulas de secundaria de los centros. Por cada ciclo temático se determinó unos objetivos, contenidos y fichas de trabajo para estimular el debate. La metodología se basó en la dinámica de grupos y se pretendía trabajar los contenidos, actitudes, valores, normas, siguiendo las directrices y orientaciones para una intervención en el ámbito escolar de García (1990), asi como para la elaboración de contenidos y fichas de trabajo (Barnett y Schueller, 2001; Pérez de Villar y Torres, 1999).

El contenido tratado se estructuró en cinco bloques temáticos:

1.- Concepto de sexualidad. Actitudes. Roles sexuales.

2.- Fisiología y anatomia. Respuesta sexual. Disfunciones sexuales. Reproducción.

3.- Cambios puberales. Adolescencia. Deseo sexual. Orientación sexual. Actitudes. Conductas sexuales y presión social.

4.- M.A.: Concepto, funciones y tipos. I.V.E: presupuestos legales. C.O.P.F. Funciones y promoción de los mismos.

5.- E.T.S. y Sida. Trarımisión, prevención y conductas de riesgo.

En cada bloque temático se utilizó una metodología de dinámica de grupos. Se inicia la sesión con un video-forum acerca del contenido de esa sesión. Los vídeos utilizados forman parte del "Método práctico de información sexual en el hogar, la escuela y la enseñanza» de 
García (1985) así como las guías didácticas publicadas para su uso, con el objeto de articular un debate acerca de los mismos. Tras el visionado se generaba un debate reflexivo acerca de los temas suscitados. Concluido el debate se recogen las ideas principales. Seguidamente trabajamos los contenidos del bloque con información al respecto. Para transmitir los contenidos se usó recursos de proyección (proyector de transparencias, diapositivas, pantalla); material propagandístico acerca de M.A., E.T.S. y sida publicados por la Generalitat Valenciana; dibujos y carteles.

Posteriormente se establecía un debate con técnicas de dinámica de grupos y se concluía con la exposición de ideas más importantes a tener en cuenta de esa sesión. Se resuelven dudas y preguntas y se promueve el que traigan escritas sus dudas anónimamente para la siguiente sesión.

Concluido el programa, a la siguiente semana, se les solicita de nuevo su colaboración voluntaria y anónima en la cumplimentación de una encuesta de opinión valorativa desde el punto de vista cualitativo del programa de educación sexual recibido. Pretendíamos conocer el grado de aceptación por los alumnos, su valoración para su formación sexual y personal, exposición de los temas expuestos y medios y si habian asimilado las conductas de prevención de riesgos más importantes. Valoración de si aún persistían dudas y preocupaciones en torno al hecho sexual.

El programa fue bien acogido por los centros educativos, pero el grado de colaboración y cooperación ha sido muy variable. Ha sido muy limitada la participación de los padres, ya que sólo en uno de los centros educativos conseguimos hacer un ciclo de formación en sexualidad para los mismos de cinco sesiones de duración; si bien es de reseñar que todos dieron su consentimiento para que sus hijos recibieran esta intervención educativa. Participaron en esta experiencia 11 colegios de un total de 19 en sus aulas naturales de $2^{2}$ de E.S.O.

\section{RESULTADOS}

Para la realización del estudio obtuvimos un total de 501 encuestas válidas, de las que el $45,30 \%$ eran de mujeres y el $54,69 \%$ eran de varones, en un rango de edad comprendido entre los 12-16 años, 
siendo la media de edad de 13.35 años y desviación típica de 1.57, perteneciendo el $68,66 \%$ de la muestra a los 13 años y el $23,35 \%$ a los 14 años, repartiéndose el resto entre las demás edades. (Tabla I).

Tabla I. Distribución de la muestra en funclón del género y la edad

\begin{tabular}{|c|cc|cc|cc|}
\cline { 2 - 7 } \multicolumn{1}{c|}{} & \multicolumn{2}{c}{ Varones } & \multicolumn{2}{c|}{ Mujeres } & \multicolumn{2}{c|}{ Total } \\
\hline EDAD & $\mathrm{n}$ & $\%$ & $\mathrm{n}$ & $\%$ & $\mathrm{n}$ & $\%$ \\
\hline 12 & 7 & $2,55 \%$ & 1 & $0,44 \%$ & 8 & $1,59 \%$ \\
13 & 180 & $65,69 \%$ & 164 & $72,24 \%$ & 344 & $68,66 \%$ \\
14 & 63 & $22,99 \%$ & 54 & $23,78 \%$ & 117 & $23,35 \%$ \\
15 & 21 & $7,66 \%$ & 7 & $3,08 \%$ & 28 & $5,58 \%$ \\
16 & 3 & $1,09 \%$ & 1 & $0,44 \%$ & 4 & $0,79 \%$ \\
\hline TOTAL & 274 & $99,98 \%$ & 227 & $99,98 \%$ & 501 & $99,97 \%$ \\
\hline
\end{tabular}

EDAD MEDIA: 13,3años

D. $T .=1,57$

En cuanto al grado de información y sus fuentes en materia sexual, el $66,26 \%$ de la muestra manifiestan haber recibido información en sexualidad, siendo el porcentaje algo mayor para las mujeres (un $70,48 \%$ ) que para los varones $(62,77 \%)$ no siendo tales diferencias significativas $\left(\chi^{2}=3.30\right.$, con $\left.2 \mathrm{~g} . \mathrm{l} ., p<0.20\right)$. La fuente principal de la que obtienen esa información es, en primer lugar, de los padres con un $26,94 \%$, seguido de los amigos con un $17,36 \%$ y medios de comunicación en tercer lugar (15,36\%). Sólo un 5,78\% acudiría al profesor en búsqueda de información. Las mujeres parecen acudir más a los padres en búsqueda de información que los varones, siendo un $30,83 \%$ frente al $23,72 \%$. No aparece diferencias significativas en función del género en esta cuestión ( $\chi^{2}=8.16$, con 7 g.l., $p<1$ ). (Figura l).

En relación a conocimientos $y$ actitudes en sexualidad valoramos que sólo un $54,89 \%$ de la muestra refieren conocer bien los genitales masculinos y un $58,48 \%$ los órganos genitales femeninos. En ambos sexos se refleja en las encuestas que necesitan ampliar conocimientos 
externos $(39,20 \%)$ frente a los varones $(25,54 \%)$ siendo tales diferencias en función del genero significativas. $\left(\chi^{2}=13.20\right.$, con $3 \mathrm{~g} . \mathrm{l}$., $p<0.01)$. No obstante, la suma de los que no saben ubicarlo con aquellos que lo hacen inadecuadamente es muy alta, un $68,25 \%$. Se observa una carencia informativa importante en cuanto al conocimiento de fisiología de sus órganos genitales. Este resultado por tanto correlaciona con las carencias de conocimientos en cuanto a la relevancia del clítoris en la satisfacción sexual de la mujer, ya que un $59,08 \%$ de la muestra manifiestan que no saben si el mismo puede producir placer en la mujer, un 4,59\% contestan negativamente y sólo un $36,32 \%$ manifiestan conocer tal hecho sexual. No aparecen diferencias significativas entre ambos sexos en este conocimiento del hecho sexual $\left(\chi^{2}=1.45\right.$, con 2 g.l., $\left.p<1\right)$. Si se aprecia un mayor porcentaje de adolescentes que manifiestan conocer los cambios puberales $(71,85 \%)$, correspondiendo el $76,21 \%$ de las mujeres que dicen conocer la acción de las hormonas sobre su cuerpo frente al $68,24 \%$ de los varones que refieren conocerlo, no alcanzando significación estadística estas diferencias de porcentajes $\left(\chi^{2}=4.62\right.$, con 2 g.l., $p<0.10$ ).

De los datos obtenidos se deduce que aunque los adolescentes reciben información de sexualidad existe un desconocimiento elevado de la fisiología y funcionamiento de sus órganos sexuales que podrían tener importantes repercusiones en la salud sexual. Puede considerarse un factor de riesgo para la salud sexual del adulto, tal es el caso de posibles disfunciones sexuales femeninas como trastornos de la excitación o anorgasmia primaria afectando de esa manera a las relaciones interpersonales, autoestima, etc. Si la fuente principal de información son los padres debemos valorar una implicación mayor de los mismos en los programas de educación sexual.

Valorando estos datos junto a las actitudes y creencias en torno a la sexualidad, de las contestaciones dadas en estos factores deducimos que en general entienden por sexualidad como relaciónafectividad, placer-diversión, y reproducción un $48,10 \%$ de la muestra, no habiendo diferencias significativas de género en cuanto a un concepto global de la misma $\left(\chi^{2}=0.15\right.$, con 1 g.l., $\left.p<1\right)$. El $24,95 \%$ de los adolescentes valoran la sexualidad como relación-afecto entre dos personas; el $11,37 \%$ como placer-diversión y un $14,57 \%$ la ven como 
factor reproductivo. Existe pues una muestra que identifica que el fin principal de la sexualidad es la reproducción. Es valorable diferencias significativas entre los géneros en el hecho que en las mujeres, un $32,59 \%$ consideran la sexualidad sólo en su aspecto afectivo frente a un $18,61 \%$ de los varones $\left(\chi^{2}=12.96\right.$, con 1 g.l., $\left.p<0.001\right)$ y en cuanto a la sexualidad como placer considerada por un $5,72 \%$ de las mujeres frente al $16,05 \%$ de los varones $\left(\chi^{2}=13.14\right.$, con 1 g.l., $\left.p<0.001\right)$.

La actitud que tienen hacia la masturbación es diferente según los sexos. Atendiendo a los datos de las encuestas, el $57,26 \%$ de las mujeres consideran que no es una práctica de interés para ellas, y sólo un $21,58 \%$ consideran que es una conducta sexual que nos produce placer, un $16,74 \%$ refieren ser una práctica sexual para conocer mejor nuestro cuerpo. Los varones parecen tener más claro que la masturbación produce placer (un 50,72\%) frente al $25,18 \%$ que refieren que no les interesa y un $16,05 \%$ que la considera adecuada para conocer mejor nuestro cuerpo. En general, existen diferencias significativas de genero en torno a este aspecto de la sexualidad $\left(\chi^{2}=65.09\right.$, con $4 \mathrm{gll}$, $p<0.001)$. La masturbación es considerada como actividad sexual placentera en mayor proporción por los varones que las mujeres, siendo estas diferencias significativas $\left(\chi^{2}=44.97\right.$, con 1 g.l., $\left.p<0.001\right)$; siendo también significativas, las diferencias en cuanto al grado de interés que muestran ante la misma, siendo en la mujer mucho menor que en los varones $\left(\dot{\sim}^{2}=53.38\right.$, con 1 g.l., $\left.p<0.001\right)$. De la muestra total se deduce que sólo un $2,59 \%$ la vivencian con culpabilidad, siendo mayor en los varones que las mujeres $\left(\chi^{2}=4.82\right.$, con $\left.1 \mathrm{g.l} ., p<0.05\right)$ y que en este rango de edad la actitud hacia la masturbación parece estar entre dos posiciones donde se distribuye casi la totalidad de la muestra: los que la consideran como placer $(37,52 \%)$ y los que no tiene interés para ellos (39,72\%). El concepto que tienen de la misma, en un $54,69 \%$ consideran que es una práctica que no perjudica nuestra salud, un $29,52 \%$ considera que nos debilita, puede provocar daños o descontrol, siendo en las chicas un $20,69 \%$ de las mismas y en varones el $36,84 \%$ $\left(\chi^{2}=27.72\right.$, con 4 g.l., $\left.p<0.001\right)$. Permanece pues un $29,52 \%$ que mantiene creencias erróneas o inexactas sobre uno de los aspectos de la sexualidad más distorsionados. Deducimos, pues, un nuevo factor de riesgo para la salud sexual, al ser identificada la misma con un concepto sexual negativo. 
Respecto a la homosexualidad, los adolescentes opinan que es una opción sexual en el $67,46 \%$, siendo un $73,56 \%$ de las mujeres quienes manifiestan tal hecho y los varones en un $62,40 \%$ alcanzando estas diferencias significación estadística $\left(\chi^{2}=7.04\right.$, con 1 g.l., $\left.p<0.01\right)$. De la muestra, opinan que se deben a un trastorno hormonal el $15,16 \%$, a una enfermedad el $12,97 \%$, y un $2,39 \%$ a la herencia, no aparece diferencias significativas entre los sexos $\left(\chi^{2}=8.03\right.$, con 4 g.l., $\left.p<0.10\right)$. Por lo que un $30,52 \%$ de los encuestados mantienen creencias erróneas en torno a la homosexualidad.

En relación a si conocen si el uso de sustancias psicoactivas pueden mejorar la capacidad sexual en una relación sexual, el 59,08\% refieren desconocer si afecta sexualmente, un $25,54 \%$ manifiestan que no aumenta nuestra capacidad sexual y un $15,36 \%$ consideran que la incrementa. No aparecen diferencias significativas en este aspecto $\left(\chi^{2}=4.44\right.$, con 2 g.l., $\left.p<0.20\right)$. Consideramos que tal desconocimiento de los efectos de las drogas estimulantes podría constituir otro factor de riesgo importante en su salud sexual ya que un $74,44 \%$ tienen o desconocimiento o ideas erróneas acerca del papel de las drogas estimulantes en la sexualidad. Por último, referir que un $7,17 \%$ de la muestra refieren haberse sentido presionados u obligados a mantener algún tipo de relación sexual, dato que debemos tener en cuenta en los programas de educación sexual con el desarrollo de habilidades de aceptación y rechazo para el establecimiento de relaciones sexuales libres y consentidas.

Como conclusión de este apartado de información, fuentes, creencias y actitudes en torno al hecho sexual destacamos la falta de conocimientos sobre el propio desarrollo sexual o el del otro sexo, de conocimientos adecuados sobre la sexualidad, y la existencia de creencias erróneas y mitos sobre la sexualidad que hacen que una buena parte de los adolescentes tengan una idea sesgada sobre cómo debe ser una relación sexual lo que dificultan el desarrollo de una sexualidad sana, placentera, saludable, y por tanto los convierte en un grupo de riesgo para el desarrollo de disfunciones sexuales entre otros.

En relación a los conocimientos de M.A., E.T.S. y Sida, los resultados nos muestran que un $59,68 \%$ de los encuestados refieren conocer que son los M.A. frente a un $40,31 \%$ que manifiestan desconocimiento en cuanto a su existencia. Las mujeres manifiestan 
conocerlos en un $61,67 \%$ y los varones suponen un $58,02 \%$. Conocen que tienen como función evitar un embarazo el $92,41 \%$ de la muestra, un $2,59 \%$ piensan que su función es abortar, el 1,99\% evitar relaciones sexuales y un $2,99 \%$ desconoce la función de los mismos, sin diferencias significativas en función del sexo $\left(\chi^{2}=5.14\right.$, con $3 \mathrm{~g} . \mathrm{l}$., $p<0.20)$. En nuestra muestra, el $91,61 \%$ manifiestan no haber utilizado ningún tipo de M.A. y un $4,19 \%$ sí lo han usado, siendo los varones en mayor proporción $(6,20 \%)$ que las mujeres $(1,76 \%)$, siendo estas diferencias significativas $\left(\chi^{2}=10.64\right.$, con 2 g.l., $\left.p<0.01\right)$. De los M.A. que enumeran conocer (pregunta de opción múltiple) en función de las respuestas agrupadas en las siguientes categorias (Figura II), es el preservativo el método más conocido, seguido de los A.O., D.I.U. e irreversibles en ambos sexos (resultado similar en esta cuestión lo encontramos en Maroto, Moreno, Rubio, Ortiz y Escobar, 1998). Tomado en su globalidad, existen diferencias de género en esta cuestión $\left(\chi^{2}=27.22\right.$, con $\left.6 \mathrm{~g} . \mathrm{l} ., p<0.001\right)$. Concluimos que parece ser que el preservativo es el método más popular.

Figura II. Métodos anticonceptivos que conocen agrupados por categorías (pregunta de opclón múltiple, $N=944$ )

\section{PORCENTAJES}

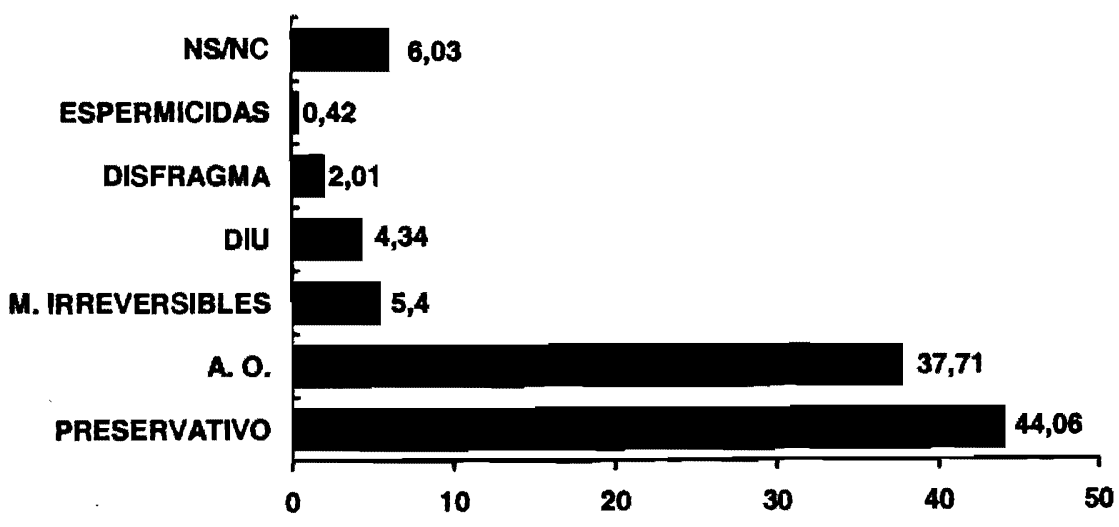


Analizando las ideas y creencias erróneas respecto al uso del mismo, vemos que los adolescentes lo consideran un método seguro en el $66,66 \%$ de la muestra y un $20,35 \%$ lo consideran inseguro con una muestra también importante del $12,97 \%$ que manifiestan desconocer su grado de seguridad. Los varones lo consideran más seguro $(73,72 \%)$ que las mujeres $(58,14 \%)$ siendo esta diferencia significativa $\left(\chi^{2}=13.65\right.$, con 2 g.l., $\left.p<0.01\right)$, lo que podría verse relacionado con el desconocimiento sobre su uso. Manifiestan saber usar correctamente un preservativo en la relación sexual el $62,67 \%$, no sabrían colocarlo adecuadamente el $21,95 \%$ y un $15,36 \%$ no lo sabe (Figura III). De ello concluimos que un $37,31 \%$ de la muestra o usarían mal el preservativo o no sabrían como utilizarlo. Los varones manifiestan un porcentaje más elevado de conocimiento sobre su uso adecuado que las mujeres un $(66,05 \%$ frente a $58,09 \%)$ manifestándose diferencias de genero significativas $\left(\chi^{2}=9.08\right.$, con 2 g.l., $\left.p<0.02\right)$. Estos resultados arrojan datos preocupantes en tanto que un porcentaje importante $(37,31 \%)$ de los adolescentes encuestados están expuestos a riesgos para su salud sexual, en cuanto a un E.M.N.D. o E.T.S.-Sida, si mantienen relaciones sexuales a estas edades $o$ en un futuro inmediato. Respecto al coito interrumpido, los adolescentes refieren que no es un método seguro en el $42,91 \%$, pero el $57,07 \%$ o bien consideran que es un M.A. seguro, o bien desconocen si lo es, siendo las mujeres quienes manifiestan mas desconocimiento que los varones (un $61,22 \%$ frente a un $53,64 \%$ ) con diferencias significativas $\left(\chi^{2}=6.66\right.$, con 2 g.l., $p<0.05$ ). Vemos que aunque nuestra muestra estudiada conozca diferentes M.A. no tienen adecuada información sobre el uso o seguridad de los mismos, sobre todo del preservativo y ello es importante de cara a riesgos para su salud sexual, dado que en muchos estudios se refleja que el preservativo era el M.A. más utilizado (Gascón, Navarro, Gascón, Pérula de Torres, Jurado y Montes, 2003b). Esto es valorable de cara a intervenciones educativas en el área sexual a edades tempranas, antes de iniciar relaciones sexuales.

Respecto a las E.T.S., los adolescentes del estudio conocen que se contagian a través de las relaciones sexuales en el $81,83 \%$, no saben tal hecho un $14,97 \%$ y un $3,19 \%$ manifiesta no ser ésta la vía de contagio, no alcanzando las diferencias entre sexos significación estadistica $\left(\chi^{2}=0.83\right.$, con 2 g.l., $\left.p<1\right)$. 
Figura III. Conocimiento del uso correcto del preservativo

PORCENTAJES

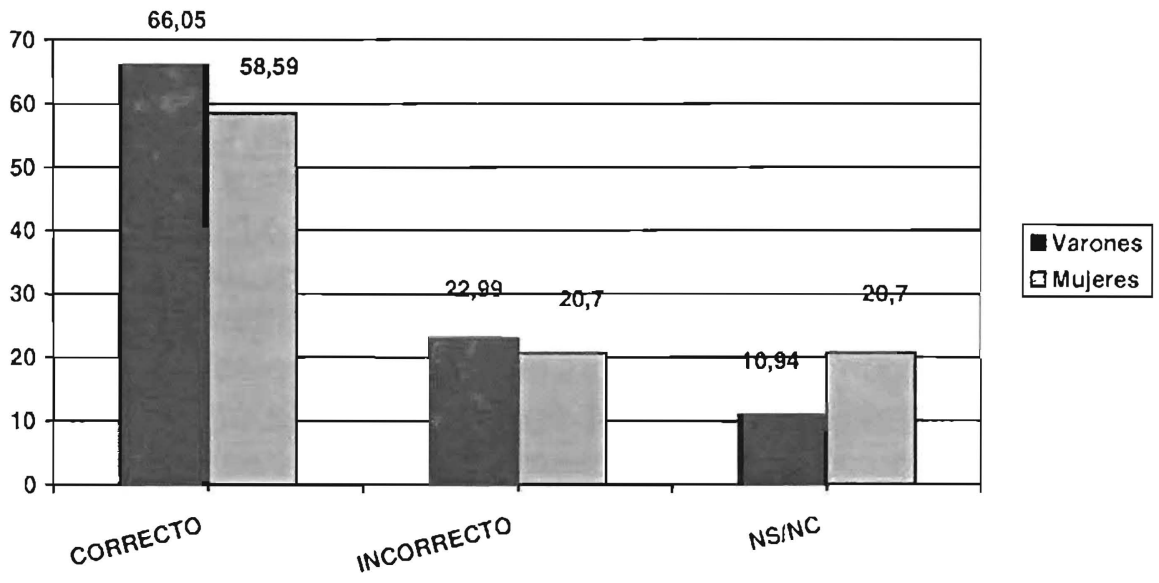

$C h r^{2}=9.08, p<0.025$

El análisis de la actividad sexual a través de los datos obtenidos de la muestra de adolescentes encuestados nos permite inferir que un $47,70 \%$ reconocian haber tenido algún tipo de actividad sexual (un $56,20 \%$ de varones frente al $37,44 \%$ de mujeres), un $47,10 \%$ no tienen actividad sexual (un $60,79 \%$ de mujeres frente al $35,76 \%$ de varones) y el $5,18 \% \mathrm{~ns} / \mathrm{nc}$. (Tabla II). Se pone de manifiesto diferencias de género significativas $\left(\chi^{2}=35.06\right.$, con 2 g.l., $\left.p<0.001\right)$. De los escolares que reconocen tener algún tipo de actividad sexual, la misma se evaluó a través de una pregunta con tres alternativas de respuesta no excluyentes: conductas autoestimulatoria, besos y caricias con el sexo contrario, y conducta sexual coital. Considerando como referencia la muestra en su globalidad ( $N=501)$, los resultados indican que un $36,32 \%$ de los escolares realizan conductas autoestimulatorias, un $41,51 \%$ caricias y besos con el sexo contrario, y un 3,39\% de la misma realizan actividad sexual coital, con diferencias significativas de genero 
$\left(\chi^{2}=31.00\right.$, con 2 g.l., $\left.p<0.001\right)$. En función del análisis por sexo, encontramos que en cualquiera de las manifestaciones sexuales, los varones presentaban mayor porcentaje. Así, analizando las diferencias significativas en función del sexo observamos que el $56,20 \%$ de varones realizan conductas autoestimulatorias frente al $12,33 \%$ de las mujeres en esta misma actividad $\left(\chi^{2}=103.29\right.$, con 1 g.l., $\left.p<0.001\right)$; las caricias y besos con el sexo contrario la mantiene el 44,89 de los varones frente a un $37,44 \%$ de mujeres $\left(\chi^{2}=2.83\right.$, con 1 g.l., $\left.p<0.10\right)$; el coito es practicado por un $4,74 \%$ de varones frente a un $1,76 \%$ de las mujeres en esta actividad sexual $\left(\chi^{2}=2.52\right.$, con 1 g.l., p-valor. 0,11 , con corrección de Yates). Se puede observar por los resultados obtenidos que los porcentajes de varones adolescentes que refieren haber tenido algún tipo de conducta sexual son mayores que el de las mujeres, pero las diferencias son significativamente estadísticas en la conducta de autoestimulación, cuando se tiene en cuenta la muestra total. (Tabla III).

De aquellos que manifiestan haber tenido actividad sexual ( $N=239)$, se observa que en las mujeres de nuestro estudio la conducta sexual más frecuente era los besos y caricias con el sexo contrario $(100 \%$ de la muestra que afirma tener experiencia sexual), la conducta autoestimulatoria en el $32,94 \%$ y la actividad coital en un $4,70 \%$. Las manifestaciones sexuales más frecuentes en los varones de la muestra que afirman tener experiencias sexuales, se observa que la conducta sexual más frecuente es la autoestimulación ( $100 \%$ de la muestra), seguida de caricias y besos con el sexo contrario $(79,87 \%)$ y actividad sexual coital en un $8,44 \%$. El análisis en función del genero nos muestra diferencias significativas en las conductas de masturbación $\left(\chi^{2}=131.94\right.$, con 1 g.l., $p$-valor. 0 , con corrección de Yates) y en la conducta sexual de besos y caricias $\left(\chi^{2}=17.91\right.$, con 1 g.l., $p<0.001$, con corrección de Yates) y no son significativas en la actividad sexual coital $\left(\chi^{2}=0.66\right.$, con 1 g.l., p-valor. 0.41 , con corrección de Yates).

En la pregunta de la encuesta que se valora el tipo de actividad sexual que han mantenido, también consideramos la edad de inicio de cada una de las conductas sexuales. Tomando como referencia la muestra que afirma tener actividad sexual ( $\mathrm{N}=239)$, el $47,70 \%$ de la muestra total, respecto de la edad de inicio podemos concluir que: en 
Tabla II. Nivel de actividad sexual de los adolescentes ( $N=501)$

\begin{tabular}{|c|cc|cc|cc|}
\cline { 2 - 7 } \multicolumn{1}{c|}{} & \multicolumn{2}{c|}{ VARONES } & \multicolumn{2}{c|}{ MUJERES } & \multicolumn{2}{c|}{ TOTAL } \\
\cline { 2 - 7 } \multicolumn{1}{c|}{} & $\mathrm{n}$ & $\%$ & $\mathrm{n}$ & $\%$ & $\mathrm{n}$ & $\%$ \\
\hline $\begin{array}{c}\text { Tienen actividad } \\
\text { sexual } \\
\text { No tienen }\end{array}$ & 154 & 56,20 & 85 & 37,44 & 239 & 47,70 \\
$\begin{array}{c}\text { actividad sexual } \\
\text { NS / NC }\end{array}$ & 22 & 85,02 & 4 & 1,76 & 26 & 5,18 \\
\hline
\end{tabular}

$C h r^{2}=35.06, p<0.001$

Tabla III. Tipo de actividad sexual de los adolescentes en funclón del sexo $(N=501)$

\begin{tabular}{|c|cc|cc|cc|cc|}
\cline { 2 - 7 } \multicolumn{1}{c|}{} & \multicolumn{2}{c|}{ TOTAL } & \multicolumn{2}{|c|}{ MUJERES } & \multicolumn{2}{|c|}{ VARONES } & \multicolumn{2}{|c|}{} \\
\cline { 2 - 7 } \multicolumn{1}{c|}{} & $\mathrm{n}$ & $\%$ & $\mathrm{n}$ & $\%$ & $\mathrm{n}$ & $\%$ & $\chi^{2}$ । gl. & $p$ \\
\hline Besos y caricias & 208 & 41,51 & 85 & 37,44 & 123 & 44,89 & 2,83 & 0,10 \\
Masturbación & 182 & 36,32 & 28 & 12,33 & 154 & 56,2 & 103,29 & 0,001 \\
Coito & 17 & 3,39 & 4 & 1,76 & 13 & 4,74 & $2,52 *$ & 0,11 \\
\hline
\end{tabular}

${ }^{\star}$ Chr con corrección de Yates

las conductas de autoestimulación las mujeres de nuestro estudio la inician entre los 9 y 15 años, correspondiendo el mayor porcentaje entre los $12-13$ años $(74,99 \%)$ con una edad media de inicio a los 12,2 años y D.T.=2. Si contrastamos con los varones, valoramos que el rango de edad se sitúa entre los 8 y 14 años, correspondiendo el mayor porcentaje entre los $11-12$ años $(54,54 \%)$, con una edad media de inicio de 11,3 años y D.T.=2.02. Las mujeres inician las conductas de autoestimulación un año más tarde que los varones $\left(\chi^{2}=19.79\right.$, con 1 g.l., $p<0.001$ ). 
Los primeros contactos sexuales interpersonales se sitúan en ambos sexos en un rango de edad que va desde los 8 a 15 años. En las mujeres, ésta parece ser la conducta sexual más frecuente, y se inicia con más frecuencia a los 12 años $(42,35 \%)$ y 13 años $(29,41 \%)$, con una edad media de inicio de 12,07 años y D.T.=2.34. En los varones, el mayor porcentaje también corresponde con los 12 años $(30,89 \%)$ y 13 años $(23,57 \%)$, siendo la edad media de inicio de sexo interpersonal de 11,5 años y D.T. $=2.29$. con diferencias significativas entre los sexos $\left(\chi^{2}=17.58\right.$, con 1 g.l., $\left.p<0.001\right)$.

Otra de las conductas sexuales evaluadas se refiere a la edad de inicio de su primera relación sexual coital. Teniendo en cuenta la muestra que afirma tener experiencia sexual, la actividad coital reconocen haberla practicado el $7,11 \%$ con una edad media de inicio de 13,8 años en nuestra muestra de escolares con relación sexual. Las mujeres reconocen esta actividad sexual en un $4,70 \%$ y la inician entre los 13-16 años correspondiendo el mayor porcentaje a los 13 años $(50 \%)$ con una edad media de inicio de 14,2 años y D.T.=1.33; los varones, por su parte, reconocen esta actividad sexual en un $8,44 \%$, y la inician entre los 11-15 años siendo el mayor porcentaje de inicio a los $14-15$ años $(69,22 \%)$ con una edad media de inicio de 13,7 años y D.T.=1.57. También en esta conducta sexual comprobamos que los varones inician antes las relaciones sexuales completas que las mujeres, y a pesar de las diferencias en porcentajes éstas no son significativas $\left(\chi^{2}=0.82\right.$, con $1 \mathrm{~g} . \mathrm{l}$., p-valor. 0.36 , con corrección de Yates). (Tablas IV y V). Este dato es relevante ya que en ambos sexos nos indica que aunque muy pequeño, un porcentaje de adolescentes inician precozmente relaciones sexuales coitales si lo comparamos con los resultados de otros estudios.

Decidimos también recoger en nuestro estudio los intereses y demandas de información de los escolares en materia de sexualidad. Tuvimos en cuenta esta cuestión en la encuesta valorando con una pregunta de respuesta abierta que posteriormente se agrupa y categoriza en bloques temáticos para su análisis. El objetivo era que incluyeran sus dudas, intereses y demandas de información que desearían que se tratara en el programa de educación sexual. Se recogieron un total de 239 preguntas o demandas que hemos agrupado en diferentes bloques temáticos con el fin de delimitar en grupos sus 
Intereses, conducta sexual y comportamientos de riesgo...

Tabla IV. Distribuclón de la muestra en función de las edades de inicio más frecuentes en las diferentes actividades sexuales en función del sexo $(N=239)$

\begin{tabular}{|c|c|c|c|c|c|c|c|c|c|}
\hline & \multicolumn{3}{|c|}{ TOTAL } & \multicolumn{3}{|c|}{ MUJERES } & \multicolumn{2}{|r|}{ VARONES } & \multirow[b]{2}{*}{$x^{2}+8.1$} \\
\hline & $\bar{n}$ & $\begin{array}{c}\text { EDAD } \\
\text { INI- } \\
\text { CIO }\end{array}$ & $\%$ & $\bar{n}$ & $\begin{array}{l}\text { EDAD } \\
\text { INI- } \\
\text { ClO }\end{array}$ & $\%$ & & $\begin{array}{l}\text { EDAD } \% \\
\text { INI- } \\
\text { Clo }\end{array}$ & \\
\hline $\begin{array}{l}\text { Masturber } \\
\text { ción }\end{array}$ & 97 & & 53.28 & 21 & $12-13$ & 74,99 & & $11-12 \quad 54,54$ & $19,79 \quad 0,001$ \\
\hline $\begin{array}{l}\text { Caricias, } \\
\text { besos sexo } \\
\text { cotrario }\end{array}$ & 128 & $12-13$ & 61,53 & 61 & $12-13$ & 71,76 & & $1 2 - 1 3 \longdiv { 5 4 , 4 6 }$ & $17,58 \quad 0,001$ \\
\hline $\begin{array}{l}\text { Actividad } \\
\text { coital }\end{array}$ & 10 & $14-15$ & 58,82 & 2 & 13 & 50 & & $1 4 - 1 5 \longdiv { 6 9 , 2 2 }$ & $0,82^{*}$ \\
\hline
\end{tabular}

*Chr con corrección de Yates

Tabla V. Medias y desviaciones típicas del inicio de las diferentes conductas sexuales por sexo

\begin{tabular}{|c|c|c|c|c|c|c|c|c|c|}
\hline & \multicolumn{3}{|c|}{ VARONES } & \multicolumn{3}{|c|}{ MUJERES } & \multicolumn{3}{|c|}{ TOTAL } \\
\hline & $\begin{array}{l}\text { Edad } \\
\text { media }\end{array}$ & D.T. & & $\begin{array}{l}\text { Edad } \\
\text { media }\end{array}$ & D.T & $\mathbf{n}$ & $\begin{array}{l}\text { Edad } \\
\text { media }\end{array}$ & D.T. & $\mathrm{n}$ \\
\hline Masturbacion & 11,3 & 2,02 & 154 & 12,2 & 2 & 28 & 11,4 & 2,29 & 182 \\
\hline $\begin{array}{l}\text { Caricias, besos } \\
\text { sexo contrario }\end{array}$ & 11,5 & 2,29 & 123 & 12,07 & 2,34 & 85 & 11,7 & 2,3 & 208 \\
\hline Coito & 13,7 & 1,57 & 13 & 14,2 & 1,33 & 4 & 13,8 & 1,73 & 17 \\
\hline
\end{tabular}

intereses. Del total de preguntas realizadas $(\mathrm{N}=239)$, la mayor parte de los encuestados les preocupa y demandan información en los siguientes bloques temáticos: en primer lugar, sobre temas relacionados con las relaciones sexuales en su vertiente tanto afectiva, relacional, y física-sexual (un 33,05\% del total de preguntas realizadas); en segundo lugar, respecto a conocimientos de fisiología, anatomía y funcionamiento de los órganos genitales $(19,65 \%)$, en tercer lugar, demandan información en materia de M.A $(15,48 \%)$, le siguen las referidas a la conducta sexual de autoestimulación $(14,64 \%)$, los aspectos reproductivos de la sexualidad $(8,39 \%)$, las E.T.S. $(5,85 \%)$; el aborto $(2,51 \%)$ y por último, la homosexualidad $(0,41 \%)$ (Figura IV). 
Figura IV. Demandas de información de los adolescentes $(N=239)$

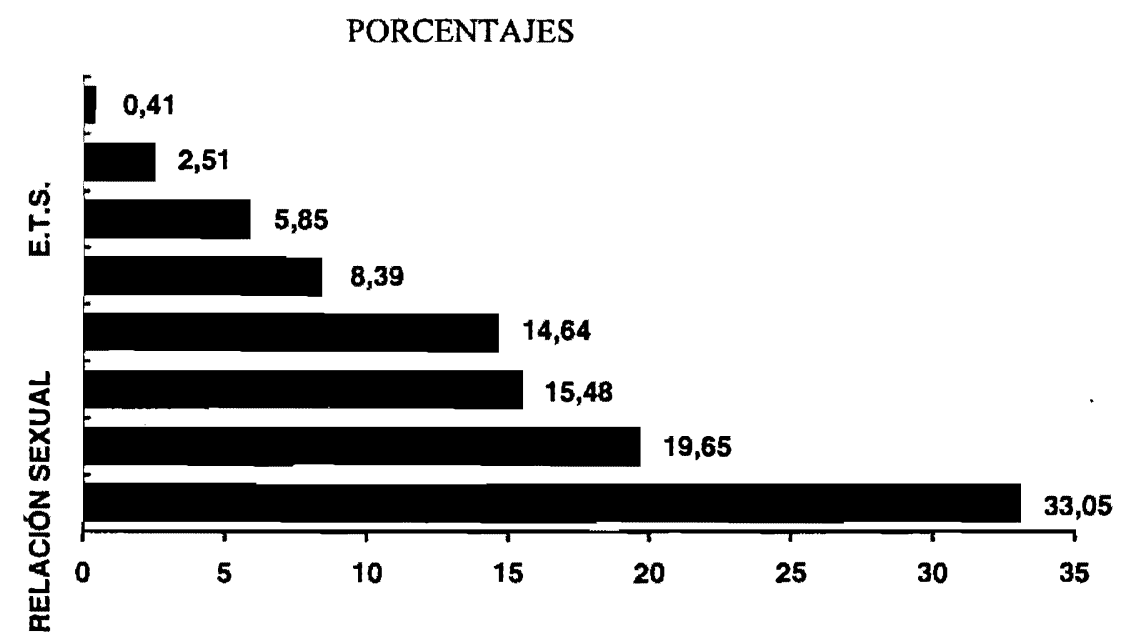

Para finalizar, valoramos el grado de información y conocimiento que tienen los encuestados respecto a los servicios sanitarios que pueden ser sus referentes en materia de información y educación sexual y donde podrían canalizar todas las cuestiones anteriormente planteadas. Se planteó una cuestión de respuesta abierta sobre qué piensan es un servicio de C.O.P.F. y para qué sirve. Un $38,32 \%$ de los encuestados no saben/no contestan a esta cuestión y el $61,67 \%$ manifiestan saberlo. Se obtiene un total de 309 respuestas que han sido agrupadas en los siguientes bloques temáticos: el $37,86 \%$ de las respuestas consideran este centro como un centro de ayuda y formación para la familia; en segundo lugar, como un centro educativo y formativo en sexualidad $(29,12 \%)$, le sigue aquellas respuestas que lo valoran como un centro que ayuda pero sin delimitar el objetivo y sin opinión clara de las funciones del mismo (18,12\%); un centro de ayuda a la sexualidad $(8,09 \%)$; sólo una minoría de las respuestas lo consideran para M.A. y evitar embarazos $(2,91 \%)$, como preventivo de E.T.S. $(2,91 \%)$, lo definen erróneamente un $0,64 \%$ y consideran que es un lugar dónde se realiza I.V.E. un $0,32 \%$ de las respuestas recogidas. Puede inferirse de estos datos que un porcentaje importante de los escolares encuestados desconocen la existencia de estos 
centros como referentes en materia de su salud sexual, y en aquellos casos que creen conocer el servicio lo conceptualizan confusamente y con referencia a aspectos muy parcializados de sus funciones.

\section{DISCUSIÓN Y CONCLUSIONES}

En el presente estudio nos planteamos analizar los comportamientos sexuales de los adolescentes, así como el grado de información en sexualidad y sus fuentes, conocimientos, creencias erróneas e intereses y demandas en sexualidad. El objetivo era analizar la muestra de adolescentes pertenecientes a nuestra área de salud con el objeto de valorar los factores de riesgo para su salud sexual así como ajustar y adecuar la intervención educativa de promoción de salud sexual a las características de los adolescentes objeto de dicha intervención, siguiendo la recomendación de Benito de la Iglesia, Paños y Pérez (1992) que sugiere que partir de un análisis de las necesidades, conocimientos, valores e intereses del alumnado incrementa la eficacia de los programas de educación sexual. Tomando en cuenta los resultados obtenidos en nuestro estudio y haciendo un breve resumen de los mismos, observamos que, en primer lugar, el perfil de las personas del estudio se corresponde con un adolescente de 13,3 años, por lo tanto concerniente a una adolescencia temprana y media. Cuando analizamos el grado de información, conocimientos y creencias erróneas, los resultados obtenidos nos permiten concluir que un $66,2 \%$ han recibido información en sexualidad y la fuente de la misma es en primer lugar de los padres, seguida de amigos, medios de comunicación, no habiendo diferencias de género significativas. Este resultado contrasta con otros estudios donde concluyen que la principal fuente de información es mayoritariamente del grupo de iguales. Ello podría deberse a que nuestra muestra es muy joven y aún tengan como referentes a sus padres por lo que sería un dato a tener en cuenta en las intervenciones educativas. Sin embargo, aunque puede estimarse este resultado como un factor positivo, valoramos de los datos obtenidos que la información recibida tiene carencias, déficits y creencias erróneas en torno al hecho sexual. Así, un 40,1\% necesita ampliar sus conocimientos de la fisiología masculina y un $36,2 \%$ de la femenina; 
el $68,25 \%$ no saben dónde se ubica el clítoris, siendo las mujeres quienes lo sitúan mejor frente a los varones (un $39,20 \%$ frente a un $25,54 \%$ ). No obstante el porcentaje es elevado y además un $59,08 \%$ desconocen que la estimulación del mismo produce placer en la mujer, no habiendo diferencias significativas entre los sexos en este hecho. Respecto al concepto de sexualidad casi la mitad de la muestra la concibe adecuadamente en un sentido global, pero aún queda un $15 \%$ que la conceptualiza sólo como factor reproductivo y es de destacar diferencias entre los sexos ya que las mujeres en un $35,59 \%$ la conciben únicamente en su vertiente afectiva frente a un $18,61 \%$ de los varones.

Mantienen ideas erróneas en torno a la masturbación un $29,52 \%$ tales como ser una práctica que nos debilita, provoca daños o descontrol, y un $54,69 \%$ tienen un concepto adecuado de la misma. Es de resaltar que el $57,26 \%$ de las mujeres consideran que es una conducta sexual que no tiene interés para ellas y sólo un $21,58 \%$ consideran que les producen placer encontrándose diferencias estadísticamente significativas en esta cuestión. Esto es importante de cara a valorar posibles riesgos de disfunciones sexuales en años venideros y que probablemente también esté asociado al dato anterior de desconocimiento tanto de la ubicación del clítoris, como de la función del mismo en la estimulación sexual, lo que puede explicar que sea una práctica sexual que carece de interés para ellas. Este dato contrasta con los varones que el $50,72 \%$ considera que es una práctica placentera. La vivencian con culpabilidad un porcentaje bajo, un $2,59 \%$.

Respecto a la homosexualidad, también se mantienen creencias erróneas en un $30,52 \%$ considerándola una enfermedad o trastorno hormonal. Tienen más claro y conocen mejor los conocimientos en torno a los cambios hormonales propios de esta edad, un $71,85 \%$. Desconocen si los efectos de las drogas estimulantes afectan a la sexualidad el $74,44 \%$.

En cuanto al conocimiento de los recursos sanitarios disponibles en torno a la sexualidad, el $38,2 \%$ desconocen este recurso y del $61,67 \%$ que cree saber las funciones de los C.O.P.F. lo conceptualizan muy confusamente. Sólo un $2,91 \%$ de las respuestas hacen relación del mismo en materia de M.A. y prevención de embarazos y un 2,91\% para prevención de E.T.S., un $0,32 \%$ para realización de la I.V.E. 
Concluimos, pues, que en materia de información y conocimientos en sexualidad presentan déficits y persisten creencias erróneas en torno a la misma, lo que en sí mismo supone un riesgo para la salud sexual al favorecer entre otras, actitudes negativas en torno al hecho sexual. Aunque reciben información sexual, la misma está sesgada o deficitaria y este dato es importante de cara a programar actividades formativas para los padres, dado que en nuestra muestra, en estas edades tempranas de la adolescencia, éstos eran el primer referente en materia sexual.

Otro dato para reflexionar y que consideramos relevante es que un elevado número de adolescentes desconocian si las drogas estimulantes afectan a la sexualidad. Este desconocimiento unido a la baja percepción del riesgo de los adolescentes y al inicio de conductas sexuales exploratorias e incipientes puede ser un riesgo para su salud sexual debido a que el uso de las mismas asociado a la actividad sexual favorecen más frecuentemente conductas sexuales sin protección adecuada, siendo un riesgo tanto para E.T.S. como E.M.N.D. (Moscoso "et al.», 1997). Otros estudios refieren que aquellos adolescentes que ingieren alcohol más frecuentemente tienen más relaciones sexuales sin protección (O»Hara «et al.», 1998).

Para finalizar este apartado debemos afirmar que tenemos que hacer un mayor esfuerzo todos los profesionales de los Centros de Salud Sexual y Reproductiva (C.S.S.R.) de cara a promover los mismos en los adolescentes ya que existen carencias informativas al respecto, no ya de las funciones del mismo sino de su misma existencia. Es importante que los adolescentes conozcan que disponen de un sistema de referencia sanitario y ello debe reflejarse con mayor relevancia en los programas de educación sexual.

En materia de anticoncepción y E.T.S. los resultados son similares en cuanto a carencias de información y déficits de la misma, dato que merece especial consideración por las consecuencias a su salud sexual. Un 40,31\% no conoce ningún método anticonceptivo, y de los que dicen conocer alguno, aparece el preservativo como el más conocido, seguido de los A.O., D.I.U. e irreversibles. Tienen claro que los M.A. evitan un embarazo (92,41\%), no han usado M.A. el $91,61 \%$ y un $4,19 \%$ han hecho uso de los mismos sobre todo los varones. Respecto a los conocimientos que tienen sobre el uso del preservativo, 
un $37,71 \%$ no sabrian usarlo correctamente y un $33,32 \%$ manifiesta que no es un método seguro o desconocen si lo es, los varones lo perciben más seguro que las mujeres (un $73,72 \%$ frente al $58,14 \%$ ) y tiene un conocimiento más adecuado del uso correcto del mismo. Un amplio porcentaje $(57,07 \%)$ considera que el "coito interruptus" es un método seguro o desconocen su grado de seguridad manifestando este desconocimiento en mayor grado las mujeres que los varones. Desconocen que las E.T.S. se contagian y transmiten por las relaciones sexuales cerca de un $20 \%$. Estas carencias informativas pueden ser riesgo para E.T.S., E.M.N.D., e I.V.E. Hemos de tener en cuenta que en nuestra muestra solo un $3,39 \%$ realizan actividad coital, pero la edad de inicio es más precoz que en otros estudios, y aunque es un porcentaje pequeño expuesto a estos riesgos, valoramos que incluso antes de estas edades es donde tenemos que hacer prevención de las mismas resolviendo no solo déficits de información sino también creencias erróneas. Usieto y Sastre (2002) refieren que aunque los adolescentes tengan información del sida mantienen creencias erróneas que les lleva a situación de riesgo. Debemos dotar de habilidades en el uso del preservativo a los adolescentes antes de que inicien actividad sexual de riesgo. Weeks, Levy, Zhu, Perhats, Handler y Flay (1995) resaltan la adecuación de los programas de educación sexual para aumentar el uso y la adecuada utilización del uso del preservativo.

Al valorar los resultados obtenidos en la actividad sexual que mantienen los adolescentes de nuestra muestra vemos que el $47,70 \%$ han tenido alguna experiencia sexual personal o interpersonal. Respecto a la práctica autoestimulatória la realizan el $36,32 \%$ de la muestra siendo mayor en los varones que en las mujeres con diferencias significativas (un $56,20 \%$ frente a un $12,32 \%$ ) con edad media de inicio en varones de 11,3 años y en la mujer de 12,2 años. En otros estudios el 47,5\% inician esta conducta antes de 12 años (Diz, Sueiro, Chas y Diéguez, 2003). Caricias y besos con el sexo contrario lo realizan el $41,51 \%$, siendo también mayor en varones que en mujeres y la edad media de inicio es de 11,5 años en varones y de 12,07 años en mujeres. La actividad coital la realizan el $3,39 \%$ siendo más frecuente en varones que en mujeres $(4,74 \%$ frente a un $1,76 \%)$ con edad media de inicio de 13,7 en varones y 14,7 en mujeres. Ballester y Gil (1994) 
establece la edad de 13-14 años para el primer coito en varones y entre 16-17 años en las mujeres.

Los resultados obtenidos muestran que la actividad sexual se produce en mayor medida en varones, con una edad media de inicio más precoz, un año antes, que en las mujeres. De aquellos que tienen relación sexual, la actividad coital representa el $7,11 \%$ siendo la edad media de 13,8 años, edad de inicio más temprana que la encontrada en otros estudios. Aunque este dato ha de tomarse con precaución dado el pequeño porcentaje que representa sobre la muestra total, lo consideramos relevante dado que representa una edad más temprana de inicio de la actividad coital en relación a otros estudios. Resultado similar lo encontramos en otro trabajo que analiza una muestra de edad similar al presente estudio, en el $7^{2}$ y $8^{2}$ grado de una escuela de Chile, situando la media de edad de inicio de coito en 12,2 años manteniéndola un $11 \%$ de la muestra (Fernández, Bustos, González, Palma, Villagran y Muñoz, 2000). Así Kapamadzija, Bjelica y Segedi (2000), la sitúan en 15,5 años; Diz "et al." (2003) en 16,8 años y la mantienen el $39,2 \%$ de la muestra; González, Rojas, Hernández y Olaiz (2005) en 15,7 años y no observa diferencias entre los sexos en muestra de adolescentes mexicanos. Otros estudios la encuadran alrededor de los 16 años (Bimbela y Cruz, 1997; Cerwonka, Isbell y Hansen, 2000; Martín y Velarde, 2001; Ramos "et al.», 2003; Gascón "et al.», 2003b). Alrededor de los 17-19 años en Sueiro, Diéguez y González, (1998), Dieguez, López y Sueiro, 1999. Por otro lado debemos decir que el porcentaje de los que tienen relaciones sexuales completas en nuestra muestra es más bajo que en otros estudios de adolescentes, que lo sitúan entre un 13\%-18\% (Martín y Velarde, 2001; Parera y Suris, 1997, Juarez, Díez, Barniol, Villamaría, Nebot y Villalba, 1999; Ras «et al.», 2004; Gónzalez «et al.», 2005; Ramos «et al.», 2003). Peltzer y Litkwa (1991) refieren que un $62 \%$ de los varones entre $12-16$ años ya tenían relación coital y un $26 \%$ de las mujeres de la misma franja de edad. Ello puede deberse al hecho de que las muestra de edades no son comparables, situándose nuestro estudio a edades más tempranas. González "et al.» (2005) refieren de los datos de su estudio, que un 2,2\% inició relación sexual coital entre 12-14 años, porcentaje bajo pero a tener en cuenta a la hora de enfocar los programas de educación sexual al seleccionar el período ternporal de intervención. 
El mayor porcentaje que tiene actividad sexual coital corresponde a los varones, aunque tomado con cautela dado la frecuencia baja de la misma en nuestra muestra. Es similar a lo encontrado en otros estudios que refieren que los varones mantienen coito más frecuentemente que las mujeres (Juarez, "et al.", 1999; Oliva «et al.", 1992; Lameiras «et al.», 2004). Siguiendo a Lameiras «et al.» (2004:73), no parece que estos datos sean explicados desde una perspectiva biológica ya que las mujeres maduran a una edad más temprana que los varones, sino más bien podria explicarse por «sus actitudes diferenciadas en la forma de entender y vivir la sexualidad (los chicos exageran sus actividades sexuales y las chicas la ocultan)"; también porque los varones sean menos selectivos en las relaciones ocasionales orientándose más al placer sexual, preocupándose más de la cantidad de relaciones mantenidas que de la calidad de esas relaciones. En el caso de las mujeres, tal y como refiere Lameiras «et al.» (2004:73) «las mujeres están más motivadas hacia relaciones de intimidad». Otros estudios que apoyan estas diferencias entre los sexos por posibles estereotipos sociales con respecto a los roles masculinos y femeninos (Oliva «et al.», 1992; Roche y Ramsbey, 1993). Por lo tanto, concluimos de nuestro estudio diferencias de género en las manifestaciones sexuales, siendo la conducta sexual del varón más precoz y frecuente, y la de las mujeres más reducida, en línea con otros resultados de estudios como en Oliva, Serra y Vallejo (1997); Ramos «et al.» (2003).

Si nos centramos ahora en las necesidades informativas de los adolescentes de nuestro estudio, podemos concluir que manifiestan o demandan información en primer lugar de las relaciones sexuales en todos sus aspectos, a continuación de conocimientos de fisiología y funcionamiento de los órganos genitales, métodos anticonceptivos, conductas autoestimulatorias, reproducción y E.T.S. en último lugar. Vemos, pues, que los contenidos referentes a riesgos para su salud sexual (tanto M.A. como E.T.S.) no son temas preferentes. Esto es importante dado que muchos estudios reflejan que la percepción del riesgo de los adolescentes en E.T.S. y Sida es baja y si además no son los temas que marcan su interés, debemos diseñar estrategias y métodos atractivos motivando que esta necesidad informativa en ellos sea prioritaria. Murphy, Rotheram-Borus y Reid, (1998) refieren que 
un $87 \%$ de los adolescentes de su estudio no se consideraban en riesgo para las mismas y Kiragu (2001) encuentra que sólo un $26 \%$ de la muestra estudiada se consideraba en riesgo, y en Lameiras, Rodríguez y Dafonte (2002) se concluye que en aquellas relaciones sexuales encuadradas dentro de una relación afectiva frente a aquellas relaciones más casuales, la percepción del riesgo es más baja.

Por último reseñar los resultados obtenidos de la valoración y percepción de los adolescentes respecto a la intervención recibida. Tales datos deben interpretarse con precaución ya que el estudio prepostintervención es sin un grupo control y los resultados obtenidos deben interpretarse con cautela. Por otro lado, el objetivo era recoger las impresiones de los adolescentes a este tipo de intervención educativa y sus valoraciones.

Analizando los datos de la evaluación cualitativa realizada postintervención, la valoración general que podemos extraer de los resultados obtenidos es que con respecto a los contenidos y temas tratados en su aspecto más formativo: un $98,49 \%$ considera que se les ha ayudado a mejorar el conocimiento de su cuerpo y un $96,76 \%$ del sexo contrario; a resolver sus dudas iniciales sobre sexualidad el $88,76 \%$ y el programa le ha facilitado la comunicación sobre sexualidad al $73,78 \%$. Con respecto a los aspectos más formales del programa, dinámica y contenidos se concluye que un $90,26 \%$ considera de su agrado los temas tratados, les han parecido adecuados la dinámica y cómo se han presentado los temas al $86,89 \%$. Los temas que más le han gustado son en primer lugar los M.A. $(55,05 \%)$ y E.T.S. $(29,96 \%)$ en segundo lugar y los que menos los temas de fisiología y adolescencia. Evalúan el programa recibido con una puntuación de 710 el $86,51 \%$ de los encuestados. Los medios utilizados que más les ha gustado son la demostración de los M.A. en primer lugar, seguido de del video-forum, transparencias expositivas del tema, le siguen los debates, trabajos en grupo y los dibujos. Por último los resultados obtenidos en cuanto a cambios en conocimientos de las conductas de prevención de riesgos: consideran al preservativo como el M.A. más adecuado a su tipo de relación si tuvieran relaciones sexuales coitales el 92,13\%; consideran que las E.T.S. se pueden contagiar en relaciones sexuales sin protección adecuada el $90,26 \%$. En caso de dudas o necesidad informativa acudirian a un C.O.P.F. el 56,92\%. 
Resultados que aunque positivos pensamos que pueden ser mejorables en futuras intervenciones así cómo los procesos de evaluación pre-postintervención de cara a obtener resultados más objetivos.

En general y a modo de conclusión de la evaluación cualitativa, podemos afirmar que los adolescentes de nuestro estudio valoran positivamente esta intervención, mejora el conocimiento de su cuerpo, les facilita la comunicación sexual y les ha ayudado a resolver las dudas iniciales planteadas; les gusta los temas tratados y los métodos utilizados para su exposición, destacando la demostración de M.A. y video-forum (este resultado lo consideramos muy positivo dado que en las demandas de información en sexualidad no eran temas prioritarios en los adolescentes). Parece que a los adolescentes les gustan los métodos participativos, dinámicos, en el sentido de discutir abiertamente sus dudas y con metodología informal y activa (Bagnall y Lockerbie, 1996; Mellanby, Phelps, Crichton y Tripp, 1995). La mayoría tienen claro que usarían el preservativo si tiene relación sexual coital y que la vía de contagio de E.T.S. son las relaciones sexuales sin protección adecuada.

En conclusión, y teniendo en cuenta los resultados obtenidos en cuanto a conductas de riesgo para la salud sexual de nuestra población objeto de estudio, se pone de manifiesto la necesidad de programas de educación sexual desde una perspectiva integral, que no sólo resuelvan déficits de información sexual, creencias erróneas o prevención de conductas de riesgo para la salud sexual, sino que favorezcan el hecho de que la sexualidad se pueda vivenciar como placentera y saludable. Diferentes estudios concluyen que si las intervenciones educativas se realizan en edades tempranas, la educación sexual iría encaminada a mejorar conocimientos más que actitudes (Juárez y Diez, 1999; Femández, Juárez y Díaz, 1999; Díez, Barniol, Nebot, Juárez, Martín y Villalbí, 1998). Consideramos importante intervenir a edades tempranas (en nuestro estudio la media de edad era de 13,2 años y un 3,39\% ya hablan tenido relación sexual coital), cuando aún los adolescentes no han tenido la primera experiencia sexual (Zoritch, Roberts y Oakley, 2000; Los jóvenes responden: diez cosas que desean los adultos sepan sobre el embarazo en la adolescencia., 2002; Consejos para evitar el embarazo 
en adolescentes, 2002; Hillman “ et al.», 1991; Lameiras "et al.», 2004), no sólo para evitar riesgos de exposición a las E.T.S., E.M.N.D. e I.V.E. (St.Lawrence, Jefferson, Alleyne y Brasfield, 1995, refieren el efecto positivo de estas intervenciones para la prevención de estas conductas de riesgos en los adolescentes); sino también otras consecuencias negativas para su sexualidad, como disfunciones psicosexuales en su vida adulta. Además, estamos de acuerdo con Lameiras "et al." (2004:74) que recomienda tal intervención desde una "perspectiva de género". Creemos que el lugar idóneo serian los centros educativos (Los jóvenes responden, 2002; Ramos "et al.», 2003; Romero de Castilla «et al.», 2001).

\section{REFERENCIAS BIBLIOGRÁFICAS}

A picture of health? A review and annotated bibliography of the health of young people in developing countries. Geneva, World Health Organization, 1995 (WHO/FHE/ADH/95.4).

Aguinaga, J., Andréu, J., Cachón, L., Comas, D., López, A. y Navarrete,

L. (2005). Informe. Juventud en España 2004. Madrid: Ministerio de Trabajo y Asuntos Sociales, Instituto de la Juventud (Injuve). Disponible en: (www.injuve.mtas.es).

Allen Meares, P. (1984). Sexually active adolescents: Implication for social work intervention and family planning services. J. Soc. Work. Hum. Sex., 3, 17-26.

Ammerman, S., Perelli, E. y Adler, N. (1992). Do adolescent understand what physicians say about sexuality and health? Clinical Pediatrics, 31(10), 590-595.

Bagnall, G. y Lockerbie, L. (1996). HIV and AIDS education for senior school pupils in Scotland: a quantitative evaluation. Health Education Journal, 55, 14.1-155.

Baldwin, T.B., Wills, A.T. y Link, B.G. (1998). Social comparison, the Threat of AIDS, and Adolescent condom use. J. Appl. Soc. Psychol., 28, 861-887.

Ballester, R. y Gil, M.D. (1994). Salud sexual (1): Análisis del comportamiento sexual de adolescentes, jóvenes y adultos en la comunidad valenciana. Análisis y Modificación de Conducta, 20(69), 111-138. 
Barnett, B. y Schueller, J. (2001). Guia para prestar servicios de salud reproductiva a los adolescentes. E.E.U.U: Family Health International.

Barros, T., Barreto, D., Pérez, F., Santander, R., Yépez, E., Abad-

Franch, F. y Aguilar, M. (2001). Un modelo de prevención primaria de las enfermedades de transmisión sexual y del VIH/SIDA en adolescentes. Rev. Panam. Salud Publica/Pan. Am. J. Public. Health, 10(2), 86-94.

Benito de la Iglesia, A., Paños, J. y Pérez, C. (1992). Intervención Comunitaria de educación para la salud para prevenir el embarazo no deseado y las enfermedades de transmisión sexual (E.T.S.) en adolescentes. Clínica y Salud, I(3), 43-65.

Bethencourt, J.M., Fumero, A., Fernández, E. y León, R. (1997). Programa de intervención comunitario para el entrenamiento de adolescentes en información y educación sexual (I): Un análisis de los primeros resultados sobre conocimientos y actitudes sexuales. Análisis y Modificación de Conducta, 28(88), 235-254.

Bimbela, J. L. y Cruz, M. T. (1997). Sida y jóvenes. La prevención de la transmisión sexual del VIH. Granada: Escuela Andaluza de Salud Pública.

Cerwonka, E. R., Isbell, T. R. y Hansen, C. E. (2000). Psychosocial factors as predictors of unsafe sexual practices among young adults. AIDS Education and Prevention, 12(2), 141-153.

Consejos para evitar el embarazo en adolescentes. The Nacional Campaign To Prevent Teen Pregnancy. Washington, DC 2002. Disponible en :http://www.teenpregnan-cy.org/resources/reading/ hispanic/espanol4.asp.

Delgado, M. (2003, julio). Fecundidad joven y adolescente. Cursos de verano de la Universidad Complutense de Madrid. Ser joven hoy: una mirada al entorno social y la sexualidad. El Escorial, Madrid.

Diéguez, J.L., López, F. y Sueiro, E. (1999). Cambios en las prácticas sexuales que conllevan riesgos de contagio (Estudio piloto). Rev. Iberoamericana de Fertilidad, 6, 57-68.

Díez, E., Barniol, J., Nebot, M., Juárez, O., Martín, M. y Villalbí, J.R. (1998). Comportamientos relacionados con la salud en estudiantes de secundaria: relaciones sexuales y consumo de tabaco, alcohol y cannabis. Gac. Sanit., 12(6), 272-280. 
Diz, M.C., Sueiro, E., Chas, M.D. y Diéguez, J.L. (2003). Comportamientos sexuales de adolescentes del medio rural gallego (3' parte). C. Med. Psicosom., 67/68, 46-60.

Ehrhardt, A., Yingling, S., Zawadski, R. y Martinez, M. (1992). Prevention of heterosexual transmission of HIV: Barriers for women. Special issue: Sexual transmission of HIV infection: Risk reduction, trauma and adaptation. Journal of Psychology and Human Sexuality, 5(12), 37-67.

Equipo DAPHNE. IV encuesta Shering sobre anticoncepción en España (2003) [datos no publicados]. Disponible en: http:// www.equipodaphne.es.

Erikson, E. H. (1993). Sociedad y adolescencia. México: Siglo XXI (14 ed.).

Erikson, E. H. (1971). Identidad, juventud y crisis. Buenos Aires: Paidós. Fernández, L., Bustos, L., González, L., Palma, D., Villagran, J. y Muñoz, S. (2000). Beliefs attitudes and knowledge about sex education. Rev. Med. Chil., 128(6), 574-583.

Fernández, S., Juárez, O. y Díaz, E. (1999). Prevención del sida en la escuela secundaria: recopilación y valoración de programas. Rev. Esp. Salud Pública, 73, 687-696.

Fisher, J. (1988). Possible effects of reference group-based social influence on AIDS-risk behavior and AIDS prevention. American Psichologist, 43, 914-920.

Franco, M.A. y Landaarroitajauregui, J.R. (2005). Sexología y salud sexual. Crítica a una salud sexual en crisis y aportaciones saludables desde la sexología. En A.L. Montejo, (Coord.), Sexualidad, Psiquiatria y cultura. (pp.89-111). Barcelona: Glosa.

García, J.L. (1985). Método práctico de información sexual en el hogar, la escuela y la enseñanza. Área informativa. Serie monográfica para adultos. Irun (Guipúzcoa): Edutest.

Garcla, J.L. (1986). Método práctico de información sexual en el hogar, la escuela y la enseñanza. Área audiovisuat: videos. Pamplona: Medusa.

García, J.L. (1990). Guia práctica de información sexual para el educador ( $2^{\mathrm{a}} \mathrm{ed}$.). Pamplona: Medusa.

Garcia, J.L. (1994). Sexualidad y adolescencia (14-17 años). Valencia: Promolibro. 
Gascón, J.A., Navarro, B., Gascón, F.J., Pérula, L.A., Jurado, A. y Montes, G. (2003a). Sexualidad y fuentes de información en población escolar adolescente. Medicina de Familia (And), 4(2), 124-129

Gascón, J.A., Navarro, B., Gascón, F.J., Pérula de Torres L.A., Jurado A. y Montes, G. (2003b). Comportamiento sexual de los escolares adolescentes en la ciudad de Córdoba. Aten. Primaria, 32(6), 355360.

Generalitat Valenciana, Conselleria de Sanidad. Serie informes de salud. Interrupciones voluntarias del embarazo en la C.V. 2004, n86. González, C., Rojas, R., Hernández, M.l. y Olaiz, G. (2005). Perfil del comportamiento sexual en adolescentes mexicanos de 12 a 19 años de edad. Resultados de la ENSA 2000. Salud pública de México, 47, 3, 209-218.

Guerra, D. (1999). Psicología de la adolescencia. En Mujer, cultura y salud: Adolescencia. Reunions Cientifiques. Serie R. № 25. (pp.8385). Valencia: Generalitat Valenciana, Conselleria de Sanitat. Dirección general de Salut Pública (Ed.).

Hillman, E., Hovell, M.F., Williams, L., Hofstetter, R., Burdyshaw, C., Rugg, D., Atkins, C., Elder, J. y Blumberg, E. (1991). Pregnancy STD and AIDS prevention: Evaluation of new image teen theatre. AIDS Educ. Prev., 3(4), 328-340.

Howard, M. (1985). Postponing sexual involvement among adolescents:

An alternative approach to prevention of sexually transmitted diseases. Journal of Adolescent Health Care, 6(4), 271-277. Juárez, O., Díez, E., Barniol, J., Villamaria, F., Nebot, M. y Villalba, J.R. (1999). Conductas preventivas de la transmisión sexual de sida, de otras infecciones y del embarazo en estudiantes de secundaria. Aten. Primaria, 24(4), 194-202.

Juárez, O. y Díez, E. (1999). Prevención del sida en adolescentes escolarizados: una revisión sistemática de la efectividad de las intervenciones. Gac. Sanit., 13(2), 150-162.

Kapamadzija, A., Bjelica, A. y Segedi, D. (2000). Sex Knowledge and behavior in male high school studentes. Med. Pregl., 53(11-12), 595-599.

Kiragu, K. (2001). Yputh and HIV/AIDS: Can we avoid catastrophe? Population Reports Series L, No. 12 Baltimore. The Johns Hopkins 
Universituy Bloomberg School of public Health Population in Formation Programs (www.infoforhealth.org/pr/prs/ sl12edsum.shtml).

Kirby, D. (1992). School-based prevention programs: Desing, evaluation, and effectiveness. En R.J. DiClemente (Ed.), Adolescents and AIS: A generation in jeopardy (pp.159-180). Newbury Park: Sage Publications.

Lameiras, M., Rodríguez, Y. y Dafonte, S. (2002). Evolución de la percepción de riesgo de la transmisión heterosexual del VIH en universitarios/as españoles/as. Psicothema, 14(2), 255-261. Lameiras, M., Rodríguez, Y., Calado, M. y González, M. (2004). Determinantes del inicio de las relaciones sexuales en adolescentes españoles. C. Med. Psicosom., 71/72, 67-75.

Lauchli, S., Heussr, R., Tschopp, A. y Gutzwiller, F. (1996). Safer sex behavior and alcohol consumption. Research Group of the Swiss HIV Prevention Study. Ann Epidemiol, 6(4), 357-364.

Lete, I. y Martínez-Etayo, M. (2004). La salud reproductiva: datos y reflexiones Gac. Sanit., 18(1), (Ejemplar dedicado a: Informe SESPAS 2004: la salud pública desde la perspectiva de género y clase social), 170-174.

Levy, S.R., Handler, A.S. y Weeks, K. (1995). Correlates of HIV risk among young adolescents in a large metropolitan midwestern epicenter. J. Sch. Health, 65, 28-32.

Loewenstein, G y Furstenberg, F. (1991). Is teenage sexual behavior rational? Journal of Applied Social Psychology, 21(12), 957-986. López, F. (1995). Educación sexual de jóvenes y adolescentes. Madrid: Siglo XXI.

Los jóvenes responden: diez cosas que desean los adultos sepan sobre el embarazo en la adolescencia. The Nacional Campaign To Prevent Teen Pregnancy. Washington, DC 2002. Disponible en: http:/ /www.teenpregnancy.org/resources/reading/hispanic/espanol4.asp Malo De Molina, C.A. (1992). Los españoles y la sexualidad. Madrid: Temas de Hoy. Maroto, A., Moreno, A., Rubio, M.M., Ortiz, C. y Escobar, F. (1998). Knowledge and use of contraceptive methods by the female population in a health-care district. Rev. Esp. de Salud Pública, 72, 547-557. 
Martín, M. y Velarde, O. (1996). Informe Juventud en España 1996. Madrid: Ministerio de Trabajo y Asuntos Sociales, Instituto de la Juventud (Injuve). Disponible en: (www.injuve.mtas.es).

Martín, M. y Velarde, O. (2001). Informe Juventud en España 2000. Madrid: Ministerio de Trabajo y Asuntos Sociales, Instituto de la Juventud. (Injuve). Disponible en: (www.injuve.mtas.es).

Martínez, J.L. (2000). Experiencias heterosexuales en la adolescencia: Implicaciones para la educación sexual. Rev. de Psa. General y Aplicada, 53, 191-209.

Masters, W.H., Johnson, V.E. y Kolodny, R.C. (1987). La sexualidad Humana. (3 vols.). Barcelona: Grijalbo.

Melchert, T. y Burnett, K.F. (1990). Attitudes, knowledge, and sexual behavior of high-risk adolescents: Implications for counseling and sexuality education. J. Couns. Dev., 68, 293-298.

Mellanby, A.R., Phelps, F.A., Crichton N.J. y Tripp, J.H. (1995). School sex education: an experimental programe with educational and medical benefit. B.M.J.; 311(12), 414-417.

Mendoza, R., Sagrera, M.R. y Batista-Foguet, J.M. (1994). Conductas de los escolares españoles relacionadas con la salud (1986-1990). Madrid: C.S.I.C.

Millstein, S.G. Petersen, A.C. y Nightingale, E.O. (1993). Promoting the health of adolescents. New directions for the twenty-first century. Nueva York: Oxford University Press.

Ministerio de Sanidad y Consumo. Datos epidemiológicos sobre la interrupción voluntaria del embarazo. Disponible en: http:// www.msc.es/.

Montero, I. y León O.G. (2002). Clasificación y descripción de las metodologías de investigación en psicología. Rev. Internacional de Psa. Clínica y de la salud/Intemational Joumal of Clinical and Health Psychology, 2, 503-508.

Moscoso, M.R. Rodríguez, L., Parrilla, I., Robles, R. y Colon, H. (1997). HIV/AIDS risk factors among adolescent students in Puerto Rico, 1994. Bol. Asoc. Med. P. R., 89(7-9), 140-145.

Mott, F.L., Fondell, M.M., Hu, P.N., Kowaleski-Jones, L. y Menaghan, E.G. (1996). The determinants of first sex by age 14 in a high-risk adolescent population. Fam. Plann. Sociology, 28(1), 13-18. Murphy, D. A., Rotheram-Borus, M. J. y Reid, H. M. (1998). Adolescent 
gender differences in HIV-related sexual risk acts, social-cognitive factors and behavioral skills. Journal of Adolescence, 21, 197-208. O'Hara, P., Parris, D., Fichtner, R. R. y Oster, R. (1998). Influence of alcohol and drug use on AIDS risk behavior among youth in dropout prevention. Joumal of Drug Education, 28(2), 159-168.

Oliva, A., Serra, L. y Vallejo, R. (1992). Conducta sexual y contraceptiva entre jóvenes andaluces. Apunt. Psicología, 35, 53-66.

Oliva, A., Serra, L. y Vallejo, R. (1993). Sexualidad y contracepción en jóvenes andaluces. Estudio cuantitativo. Sevilla: Junta de Andalucía. Consejería de Salud.

Oliva, A., Serra, L. y Vallejo, R. (1997). Patrones de comportamiento sexual y contraceptivo durante la adolescencia. Infanc. Aprend., 77, 19-34.

ONUSIDA. 2002. Informe sobre la epidemia mundial del VIH/SIDA ONU/OMS (www.unaids.org)

Organización Panamericana de la Salud. (1995). Salud de los adolescentes. Washington, DC: OPS/OMS.

Organización Panamericana de la Salud. (1998). Plan de acción de desarrollo y salud de adolescentes y jóvenes en las Américas, 1998-2001. Washington, DC: OPS/OMS.

Palau, M. (1992). Embarazo en la adolescencia. Atención Primaria, 9, 284-286.

Parera, N. y Suris, J.C. (1997). Sexuality and contraception in adolescents from Barcelona, Spain. J. Pediatr. Adolesc. Ginecol., 10, 153-157.

Peltzer, K. y Litkwa, R. (1991). Attitude towards sexual behaviour and sexual education among primary school children in Lusaka. Joumal of Psychology in Africa, 1(4), 65-71.

Pérez de Villar, M.J. y Torres, C. (1999). Dinámica de grupos en formación de formadores: casos prácticos. Barcelona: Ed. Herder (Biblioteca de Psicología).

Pick, S., Givaudan, M. y Saldívar-Garduño, A. (1994). La importancia de los factores psicosociales en la educación sexual de los adolescentes. Perinatol. Reprod. Hum., 2, 143-150.

Population and Development, I: Programme of Action adopted at the International Conference on Population and Development, Cairo 513 September 1994. New York, United Nations, Department for Economic and social Information and policy Analysis, 1995. 
Portella, E. y Castillo, A. (1988). Aspectos sociales del embarazo en adolescentes. Gaceta sanitari, 2, 150-153.

Ramos, M.T. y Cantú, P.C. (2003). EI VIH/SIDA y la adolescencia.

Revista de salud pública y nutrición, Respyn, 4(4). Disponible en: (www.uanl.mx/publicaciones/respyn).

Ramos, M., Fuertes, A., Martínez, J.L. y Hernández, A. (2003).

Comportamientos y actitudes sexuales de los adolescentes de Castilla y León. Análisis y Modificación de Conducta, 29(124), 213-238.

Ras, E., Lluis, M., Subirats, R., Pellejo, M.L., Lara, A. y Rodríguez, M.V. (2004). La educación sexual en los adolescentes desde la atención primaria. Salud Rural, XXI (3), 89-96.

Rebull, J., Reverté, M., Piñas, I., Ortí, A., González, L. y Contreras, E. (2003). Evaluación pre-post de una actividad preventiva de la infección por $\mathrm{VIH}$ dirigida a adolescentes de las comarcas del sur de Tarragona. Rev. Esp. Salud Pública, 77, 373-382.

Roche, J.P. y Ramsbey, T.W. (1993). Premarital sexuality: A five-year follow-up study of attitudes and behavior by dating stage. Adolescence, 28, 67-80.

Romero de Castilla, R.J., Lora, M.N. y Cañete, R. (2001). Adolescentes y fuentes de información de sexualidad: preferencias y utilidad percibida. Aten. Primaria, 27, 12-17.

Ruiz, M.A., Almenara, J., Rodríguez, A. y Fernández, J.R. (1990). Uso de métodos anticonceptivos en población adolescente. Rev. Esp. Pediatr., 46(3), 229-232.

Siegel, D.M., Aten, M.J., Roghmann, K.J. (1998). Self-reported honesty among middle and high school students responding to a sexual behavior questionare. J. Adolesc. Health, 23, 20-28. [Medline]

St. Lawrence, J.S., Jefferson, K.W., Alleyne, E. y Brasfield, T.L. (1995). Comparison of Education Versus Behavioral Skills Training Interventions in Lowering Sexual HIV-Risk Behavior of SubstanceDependent Adolescentes. Journal of Consulting and Clinical Psychology, 63(1), 154-157.

Stassen, K. y Thompson, R. (2000). Psicología del desarrollo: Infancia y adolescencia. México: Ed. Medica Panamericana.

Sueiro, E. Diéguez, J.L. y González A. (1998). Jóvenes que realizan estudios universitarios: salud sexual y reproductiva. Aten. Primaria, $21,283-288$. 
Usieto, R. y Sastre, J. (2002). La información y educación sobre el sida como estrategia de prevención en los jóvenes. Una experiencia entre los adolescentes escolarizados de Cantabria. Revista Española de Drogodependencias, 27(3), 445-465.

Valerio, A. (1985). Sex education program evaluations: A review and analysis. Resumen tesis doctoral. SUNY y Buffalo.

Vallés, A. (1986). Embarazo en la adolescente. Factor de riesgo. Neonatal Arch. Pediatr., 36, 127-134.

Weeks, K., Levy, S.R., Zhu, C., Perhats, C., Handler, A. y Flay, B.R. (1995). Impact of a school-based Aids prevention program on young adolescents self-efficacy skills. Health Education Research, 10(3), 329-344.

Yamada, D.J., Herrera, M., Cota, F., Núñez, M. y Guzmán, R. (2002). Salud sexual y reproductiva en adolescentes del área rural: Eficacia de la capacitación. Rev. Med. IMSS, 40(2), 137-143.

Zoritch, B., Roberts, I. y Oakley, A. (2000). Day care for preschool children. Cochrane Database Sys Rev, (2):CD000564. 